\title{
MINI-WALLS FOR BRIDGELAND STABILITY CONDITIONS ON THE DERIVED CATEGORY OF SHEAVES OVER SURFACES*
}

\author{
JASON LO ${ }^{\dagger}$ AND ZHENBO QIN ${ }^{\dagger \ddagger}$
}

\begin{abstract}
For the derived category of bounded complexes of sheaves on a smooth projective surface, Bridgeland [Bri2] and Arcara-Bertram [ABL] constructed Bridgeland stability conditions $\left(Z_{m}, \mathcal{P}_{m}\right)$ parametrized by $m \in(0,+\infty)$. In this paper, we show that the set of mini-walls in $(0,+\infty)$ of a fixed numerical type is locally finite. In addition, we strengthen a result of Bayer [Bay] by proving that the moduli of polynomial Bridgeland semistable objects of a fixed numerical type coincides with the moduli of $\left(Z_{m}, \mathcal{P}_{m}\right)$-semistable objects whenever $m$ is larger than a universal constant depending only on the numerical type. We further identify the moduli of polynomial Bridgeland semistable objects with the Gieseker/Simpson moduli spaces and the Uhlenbeck compactification spaces.
\end{abstract}

Key words. Walls, Bridgeland stability, polynomial stability, derived category.

AMS subject classifications. Primary: 14D20; Secondary: 14F05, 14J60.

1. Introduction. Since the appearance of Bridgeland's seminal work [Bri1], there have been intensive investigations of Bridgeland stability conditions on triangulated categories, which can be viewed as a mathematical approach to understand Douglas' work [Dou] on $\Pi$-stability for D-branes in string theory. Bridgeland stability conditions for smooth projective curves were classified by Macri [Mac] and Okada [Oka]. Bridgeland stability conditions on smooth projective surfaces were constructed by Bridgeland [Bri2] and Arcara-Bertram [ABL], and the topology of the stability manifolds for generic K3 categories was obtained by Huybrechts, Macri and Stellari [HMS]. Toda [Tod2] studied Bridgeland stability conditions for Calabi-Yau fibrations. A gluing procedure for Bridgeland stability conditions was found by Collins and Polishchuk [CP]. In another direction, Bayer [Bay] (see also Toda [Tod3]) defined polynomial Bridgeland stability for normal projective varieties of any dimension. The polynomial Bridgeland stability may be viewed as the large volume limit of the Bridgeland stability. The moduli stacks of Bridgeland semistable objects were investigated in [Ina, Lie, Tod1], while the moduli stacks of polynomial Bridgeland semistable objects were investigated in [Lo1, Lo2, Lo3, LQ].

The concepts of walls and chambers for Gieseker stability were introduced in [Qin] and played an important role in understanding Donaldson polynomial invariants of certain surfaces. Walls and chambers in the space of Bridgeland stability conditions are closely related to the wall-crossing phenomena discussed by Kontsevich and Soibelman $[\mathrm{KS}]$. Let $X$ be a smooth projective surface, and let $\mathcal{D}^{b}(X)$ be the derived category of bounded complexes of coherent sheaves on $X$. When $X$ is a $K 3$ or abelian surface, Bridgeland [Bri2] proved that the set of walls in the space of Bridgeland stability conditions on $\mathcal{D}^{b}(X)$ is locally finite. Whether the same conclusion holds for a general surface $X$ remains to be open.

In this paper, we analyze the set of mini-walls and mini-chambers in the space of Bridgeland stability conditions. To state our results, we introduce some notations and definitions (see Notation 2.5 and Definition 3.2 below for details). The numerical type of an object $E \in \mathcal{D}^{b}(X)$ is defined to be $\mathfrak{t}(E)=\left(\operatorname{rk}(E), c_{1}(E), c_{2}(E)\right)$. Fix

\footnotetext{
*Received March 9, 2012; accepted for publication February 1, 2013

${ }^{\dagger}$ Department of Mathematics, University of Missouri, Columbia, MO 65211, USA (\{locc; qinz $\}$ missouri.edu).

‡Partially supported by an NSF grant and by a grant from the Simons Foundation.
} 
$\beta, \omega \in \operatorname{Num}(X)_{\mathbb{Q}}$ with $\omega$ being ample, and fix a numerical type $\mathfrak{t}=\left(r, c_{1}, c_{2}\right)$. Bridgeland [Bri2] and Arcara-Bertram [ABL] constructed Bridgeland stability conditions $\left(Z_{m}, \mathcal{P}_{m}\right)$ parametrized by $m \in(0,+\infty)$. Regard $(0,+\infty)$ as a subset in the space of Bridgeland stability conditions. Then walls and chambers in $(0,+\infty)$ are referred to as mini-walls and mini-chambers of type $(\mathfrak{t}, \beta, \omega)$.

TheOREM 1.1. Let $\beta, \omega \in \operatorname{Num}(X)_{\mathbb{Q}}$ with $\omega$ being ample, and let $\mathfrak{t}=\left(r, c_{1}, c_{2}\right)$.

(i) The set of mini-walls of type $(\mathfrak{t}, \beta, \omega)$ in $(0,+\infty)$ is locally finite.

(ii) There exists a positive number $\widetilde{M}$, depending only on $\mathfrak{t}, \omega$ and $\beta$, such that there is no mini-wall of type $(\mathfrak{t}, \beta, \omega)$ in $[\widetilde{M},+\infty)$.

Theorem 1.1 has been observed in the special case considered in Sect. 4 of [ABL]. Moreover, Theorem 1.1 (ii) strengthens the Proposition 4.1 in [Bay] (see Lemma 2.6 below). In fact, we prove in Theorem 4.4 that whenever $m \geq \widetilde{M}$, an object $E \in \mathcal{D}^{b}(X)$ with $\mathfrak{t}(E)=\mathfrak{t}$ is $\left(Z_{m}, \mathcal{P}_{m}\right)$-semistable if and only if $E$ is $\left(Z_{\Omega}, \mathcal{P}_{\Omega}\right)$-semistable. Here $\Omega=(\omega, \rho, p, U)$ is the stability data from Subsect. 2.2 , and $\left(Z_{\Omega}, \mathcal{P}_{\Omega}\right)$ denotes the polynomial Bridgeland stability constructed in [Bay].

The main idea in proving Theorem 1.1 (i) is to find an upper bound for $\operatorname{rk}(A)$ if $A$ defines a mini-wall of type $(\mathfrak{t}, \beta, \omega)$ and if the mini-wall is contained in an interval $I=[a,+\infty)$. This upper bound is universal in the sense that it depends only on $I$ and $(\mathfrak{t}, \beta, \omega)$. Combining this idea with an expanded version of the proof of the Proposition 4.1 in [Bay] also leads to the proof of Theorem 1.1 (ii).

Next, we classify all the polynomial Bridgeland semistable objects in terms of Gieseker/Simpson semistable sheaves. Let $\overline{\mathfrak{M}}_{\Omega}(\mathfrak{t})$ be the set of all $\left(Z_{\Omega}, \mathcal{P}_{\Omega}\right)$-semistable objects $E \in \mathcal{P}_{\Omega}((0,1])$ with $\mathfrak{t}(E)=\mathfrak{t}$. Let $\overline{\mathfrak{M}}_{\omega}(\mathfrak{t})$ be the moduli space of sheaves $E \in \operatorname{Coh}(X)$ which are Simpson-semistable with respect to $\omega$ and satisfy $\mathfrak{t}(E)=\mathfrak{t}$. For $r>0$, define $\mathfrak{M}_{\omega}(\mathfrak{t})$ be the moduli space of locally free sheaves $E$ which are $\mu_{\omega}$-stable and satisfy $\mathfrak{t}(E)=\mathfrak{t}$, and define $\overline{\mathfrak{U}}_{\omega}(\mathfrak{t})$ to be the Uhlenbeck compactification space associated to $\omega$ and $\mathfrak{t}$. The case when $r=0$ is covered by Lemma 2.10 and Lemma 2.11. For $r \neq 0$, we have the following.

THEOREM 1.2. Let $\Omega=(\omega, \rho, p, U)$ be from Subsect. 2.2 with $\omega \in \operatorname{Num}(X)_{\mathbb{Z}}$. Fix a numerical type $\mathfrak{t}=\left(r, c_{1}, c_{2}\right)$ with $\operatorname{gcd}\left(r, c_{1} \omega\right)=1$. Let $\tilde{\mathfrak{t}}=\left(-r, c_{1}, c_{1}^{2}-c_{2}\right)$.

(i) If $r>0$, then $\overline{\mathfrak{M}}_{\Omega}(\mathfrak{t}) \cong \overline{\mathfrak{M}}_{\omega}(\mathfrak{t})$.

(ii) If $r<0$ and $c_{1} \omega / r<\beta \omega$, then $\overline{\mathfrak{M}}_{\Omega}(\mathfrak{t}) \cong \overline{\mathfrak{M}}_{\omega}(\tilde{\mathfrak{t}})$.

(iii) If $r<0$ and $c_{1} \omega / r=\beta \omega$, then $\overline{\mathfrak{M}}_{\Omega}(\mathfrak{t}) \cong \overline{\mathfrak{U}}_{\omega}(\tilde{\mathfrak{t}})$.

We therefore have a complete description of the moduli spaces of $\left(Z_{\Omega}, \mathcal{P}_{\Omega}\right)$ semistable objects on every smooth projective surface. In view of Theorem 4.4 , we obtain a complete description of the moduli spaces of semistable objects with respect to certain Bridgeland stabilities on a smooth projective surface. We remark that similar results in the context of Bridgeland stability have been observed and studied by Bridgeland [Bri2], Kawatani [Kaw], Ohkawa [Ohk], Toda [Tod1] and Yoshioka [Yos1, Yos2, Yos3]. Similar results in the context of polynomial Bridgeland stability have also appeared in Sect. 4 of [LQ].

This paper is organized as follows. In Sect. 2, we recall the constructions of Bridgeland, Arcara-Bertram and Bayer. Theorem 1.1 (i) and (ii) are proved in Sect. 3 and Sect. 4 respectively. In Sect. 5, we verify Theorem 1.2.

Conventions. The $i$-th cohomology of a sheaf $E$ on a variety $X$ is denoted by $H^{i}(X, E)$, and its usual dual sheaf $\mathcal{H o m}\left(E, \mathcal{O}_{X}\right)$ is denoted by $E^{*}$. The derived category of bounded complexes of coherent sheaves on $X$ is denoted by $\mathcal{D}^{b}(X)$. The 
$i$-th cohomology sheaf of an object $E \in \mathcal{D}^{b}(X)$ is denoted by $\mathcal{H}^{i}(E)$, and the derived dual of $E$ is denoted by $E^{\vee}=\mathbb{R} \mathcal{H} \operatorname{com}\left(E, \mathcal{O}_{X}\right) \in \mathcal{D}^{b}(X)$.

Acknowledgment. The authors thank Professors Jun Li and Wei-Ping Li for valuable helps and stimulating discussions. The authors also thank the referee for making valuable comments and suggestions.

\section{Preliminaries.}

2.1. Constructions of Bridgeland and Arcara-Bertram. Let $X$ be a smooth complex projective surface.

Definition 2.1. Let $\omega \in \operatorname{Num}(X)_{\mathbb{R}}$ be ample, and let $v \in \mathbb{R}$.

(i) Define $\mathcal{T}_{(\omega, v)}$ to be the full subcategory of $\operatorname{Coh}(X)$ generated by torsion sheaves and torsion free $\mu_{\omega}$-stable sheaves $A$ with $\mu_{\omega}(A)>v$.

(ii) Define $\mathcal{F}_{(\omega, v)}$ to be the full subcategory of $\operatorname{Coh}(X)$ generated by torsion free $\mu_{\omega}$-stable sheaves $A$ with $\mu_{\omega}(A) \leq v$.

(iii) Define $\mathcal{A}_{(\omega, v)}^{\sharp}$ to be the abelian category obtained from $\operatorname{Coh}(X)$ by tilting at the torsion pair $\left(\mathcal{T}_{(\omega, v)}, \mathcal{F}_{(\omega, v)}\right)$, i.e., $\mathcal{A}_{(\omega, v)}^{\sharp}$ consists of all the objects $E \in$ $\mathcal{D}^{b}(X)$ satisfying the conditions:

(2.1) $\quad \mathcal{H}^{-1}(E) \in \mathcal{F}_{(\omega, v)}, \quad \mathcal{H}^{0}(E) \in \mathcal{T}_{(\omega, v)}, \quad \mathcal{H}^{i}(E)=0$ for $i \neq-1,0$.

The following lemma will be used in Case 3 in the proof of Lemma 4.3 below.

Lemma 2.2. Let $Q$ be a 0 -dimensional torsion sheaf, and $\mathcal{C} \in \mathcal{T}_{(\omega, v)}$. If $\mathcal{G}$ sits in an exact sequence $0 \rightarrow \mathcal{G} \rightarrow \mathcal{C} \rightarrow Q \rightarrow 0$ of coherent sheaves, then $\mathcal{G} \in \mathcal{T}_{(\omega, v)}$.

Proof. Let $\operatorname{Tor}(\mathcal{C})$ denote the torsion subsheaf of $\mathcal{C}$. Let

$$
\operatorname{Tor}(\mathcal{C})=\mathcal{C}_{0} \subset \mathcal{C}_{1} \subset \ldots \subset \mathcal{C}_{n}=\mathcal{C}
$$

be the usual HN-filtration of $\mathcal{C}$ with respect to $\mu_{\omega}$. Let $\mu_{i}=\mu_{\omega}\left(\mathcal{C}_{i} / \mathcal{C}_{i-1}\right)$. Then for $i=1, \ldots, n$, the sheaf $\mathcal{C}_{i} / \mathcal{C}_{i-1}$ is torsion free and $\mu_{\omega}$-semistable. Moreover, $\mu_{1}>\ldots>$ $\mu_{n}$. By the definition of $\mathcal{T}_{(\omega, v)}, \mu_{1}>\ldots>\mu_{n}>v$. For $i=0,1, \ldots, n$, let $\mathcal{G}_{i}$ and $\mathcal{Q}_{i}$ be the kernel and image of the induced map $\mathcal{C}_{i} \rightarrow Q$ respectively. Then $\mathcal{G}_{i-1}=\mathcal{G}_{i} \cap \mathcal{C}_{i-1}$. The injection $0 \rightarrow \mathcal{G}_{i} / \mathcal{G}_{i-1} \rightarrow \mathcal{C}_{i} / \mathcal{C}_{i-1}$ implies that $\mathcal{G}_{i} / \mathcal{G}_{i-1}$ is torsion free. Also, we have commutative diagram of sheaves

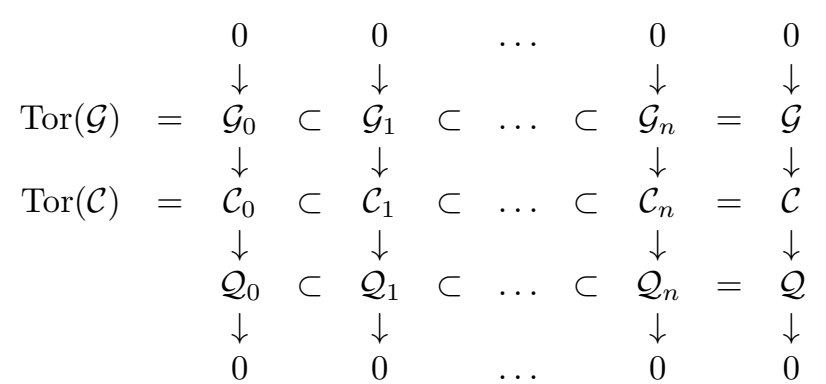

from which we obtain two exact sequences for each $i=1, \ldots, n$ :

$$
\begin{aligned}
& 0 \rightarrow \mathcal{G}_{i} / \mathcal{G}_{i-1} \quad \rightarrow \quad \mathcal{C}_{i} / \mathcal{G}_{i-1} \quad \rightarrow \quad \mathcal{Q}_{i} \quad \rightarrow \quad 0 \\
& 0 \quad \rightarrow \quad \mathcal{Q}_{i-1} \quad \rightarrow \quad \mathcal{C}_{i} / \mathcal{G}_{i-1} \quad \rightarrow \quad \mathcal{C}_{i} / \mathcal{C}_{i-1} \rightarrow 0 .
\end{aligned}
$$


Since $\mathcal{G}_{i} / \mathcal{G}_{i-1}$ is torsion free and $\mathcal{Q}_{i-1}$ is torsion, we get an exact sequence

$$
0 \rightarrow \mathcal{G}_{i} / \mathcal{G}_{i-1} \rightarrow \mathcal{C}_{i} / \mathcal{C}_{i-1} \rightarrow \widetilde{\mathcal{Q}}_{i} \rightarrow 0
$$

where $\widetilde{\mathcal{Q}}_{i}$ is a 0-dimensional torsion sheaf. Thus, $\mu_{\omega}\left(\mathcal{G}_{i} / \mathcal{G}_{i-1}\right)=\mu_{\omega}\left(\mathcal{C}_{i} / \mathcal{C}_{i-1}\right)=\mu_{i}>$ $v$, and $\mathcal{G}_{i} / \mathcal{G}_{i-1}$ is $\mu_{\omega}$-semistable. Hence

$$
\operatorname{Tor}(\mathcal{G})=\mathcal{G}_{0} \subset \mathcal{G}_{1} \subset \ldots \subset \mathcal{G}_{n}=\mathcal{G}
$$

is the usual HN-filtration of $\mathcal{G}$ with respect to $\mu_{\omega}$, and $\mathcal{G} \in \mathcal{T}_{(\omega, v)}$. $\square$

Let $\mathbf{u} \in \mathcal{N}(X) \otimes_{\mathbb{Z}} \mathbb{C}$. Define the central charge $Z_{\mathbf{u}}$ on $\mathcal{D}^{b}(X)$ by

$$
Z_{\mathbf{u}}(E)=-\int_{X} \mathbf{u} \cdot \operatorname{ch}(E) .
$$

The following lemma is due to Bridgeland [Bri2] and Arcara-Bertram [ABL].

Lemma 2.3. Let $\mathbf{u}=e^{-(\beta+i \omega)}$ where $\beta, \omega \in \operatorname{Num}(X)_{\mathbb{R}}$ and $\omega$ is ample. Then $\left(Z_{\mathbf{u}}, \mathcal{A}_{(\omega, \beta \omega)}^{\sharp}\right)$ induces a Bridgeland stability condition $\left(Z_{\mathbf{u}}, \mathcal{P}_{\mathbf{u}}\right)$ on $\mathcal{D}^{b}(X)$.

2.2. Polynomial stability and large volume limits. Let $\Omega=(\omega, \rho, p, U)$ be the stability data defined by the following:

- $\omega \in \operatorname{Num}(X)_{\mathbb{R}}$ is ample,

- $\rho=\left(\rho_{0}, \rho_{1}, \rho_{2}\right)$ with $\rho_{i}=-(-i)^{d} / d$ !,

- $p:\{0,1,2\} \rightarrow \mathbb{Z}$ is the perversity function $p(d)=-\lfloor d / 2\rfloor$,

- $U=e^{-\beta}$ for some $\beta \in \operatorname{Num}(X)_{\mathbb{R}}$.

Let $Z_{\Omega}: K\left(\mathcal{D}^{b}(X)\right)=K(X) \rightarrow \mathbb{C}[m]$ be the central charge defined by

$$
Z_{\Omega}(E)(m)=\int_{X} \sum_{d=0}^{2} \rho_{d} \omega^{d} m^{d} \cdot \operatorname{ch}(E) \cdot U=-\int_{X} e^{-(\beta+i m \omega)} \cdot \operatorname{ch}(E) .
$$

By [Bay], $Z_{\Omega}(E)(m)$ induces a polynomial stability condition $\left(Z_{\Omega}, \mathcal{P}_{\Omega}\right)$ on $\mathcal{D}^{b}(X)$.

Lemma 2.4. (Lemma 4.2 in [Bay]) We have $\mathcal{P}_{\Omega}((0,1])=\mathcal{A}_{(\omega, \beta \omega)}^{\sharp}$. In fact, if $E \in \mathcal{P}_{\Omega}((0,1]) \subset \mathcal{D}^{b}(X)$ is $\left(Z_{\Omega}, \mathcal{P}_{\Omega}\right)$-semistable, then $E$ is one of the following:

(i) $E$ is a torsion sheaf;

(ii) $E$ is a torsion free $\mu_{\omega}$-semistable sheaf with $\mu_{\omega}(E)>\beta \omega$.

(iii) $\mathcal{H}^{-1}(E)$ is a torsion free $\mu_{\omega}$-semistable sheaf with $\mu_{\omega}\left(\mathcal{H}^{-1}(E)\right) \leq \beta \omega, \mathcal{H}^{0}(E)$ is a 0 -dimensional torsion, and all other cohomology sheaves of $E$ vanish.

Notation 2.5. Fix $\mathbf{u}=e^{-(\beta+i \omega)}$. Put $\mathbf{u}_{m}=e^{-(\beta+i m \omega)}$ and

$$
Z_{m}(E)=Z_{\mathbf{u}_{m}}(E)=-\int_{X} e^{-(\beta+i m \omega)} \cdot \operatorname{ch}(E)=Z_{\Omega}(E)(m) .
$$

Let $\left(Z_{m}, \mathcal{P}_{m}\right)$ denote the Bridgeland stability condition on $\mathcal{D}^{b}(X)$ induced by $Z_{m}$.

Lemma 2.6. (Proposition 4.1 in [Bay]) Let notations be as above. Assume further that $\omega \in \operatorname{Num}(X)_{\mathbb{Q}}$. Then, an object $E \in \mathcal{D}^{b}(X)$ is $\left(Z_{\Omega}, \mathcal{P}_{\Omega}\right)$-semistable if and only if $E$ is $\left(Z_{m}, \mathcal{P}_{m}\right)$-semistable for $m \gg 0$. Moreover, for an arbitrary object $E \in \mathcal{D}^{b}(X)$, the $H N$-filtration of $E$ with respect to $\left(Z_{\Omega}, \mathcal{P}_{\Omega}\right)$ is identical to the HN-filtration of $E$ with respect to $\left(Z_{m}, \mathcal{P}_{m}\right)$ for $m \gg 0$. 
Definition 2.7. For $E \in \mathcal{D}^{b}(X)$, define its numerical type $\mathfrak{t}(E)$ by

$$
\mathfrak{t}(E)=\left(\operatorname{rk}(E), c_{1}(E), c_{2}(E)\right) .
$$

REMARK 2.8. A straight-forward computation from (2.3) shows that

$$
Z_{\Omega}(E)(m)=\operatorname{rk}(E) \omega^{2} \cdot \frac{m^{2}}{2}+i\left(c_{1}(E) \cdot \omega-\operatorname{rk}(E) \beta \omega\right) m+c(E)
$$

where

$$
c(E)=-\operatorname{ch}_{2}(E)+c_{1}(E) \cdot \beta-\operatorname{rk}(E) \cdot \frac{\beta^{2}}{2} \in \mathbb{R} .
$$

It follows that if $E, B \in \mathcal{P}_{\Omega}((0,1])=\mathcal{A}_{(\omega, \beta \omega)}^{\sharp}$ and $m>0$, then $\phi\left(Z_{\Omega}(E)(m)\right)>$ $\phi\left(Z_{\Omega}(B)(m)\right)$ is equivalent to $\operatorname{Im}\left(\overline{Z_{\Omega}(E)(m)} \cdot Z_{\Omega}(B)(m)\right)<0$, i.e.,

$$
\begin{aligned}
& \frac{\omega^{2} m^{2}}{2}\left(\operatorname{rk}(E) c_{1}(B) \omega-\operatorname{rk}(B) c_{1}(E) \omega\right) \\
< & c(B)\left(c_{1}(E) \omega-\operatorname{rk}(E) \beta \omega\right)-c(E)\left(c_{1}(B) \omega-\operatorname{rk}(B) \beta \omega\right) .
\end{aligned}
$$

\subsection{Moduli spaces, walls and chambers.}

Definition 2.9. Let notations be as above. Fix a numerical type $\mathfrak{t}=\left(r, c_{1}, c_{2}\right)$.

(i) Let $E \in \mathcal{P}_{\Omega}((0,1])=\mathcal{A}_{(\omega, \beta \omega)}^{\sharp}$ be $\left(Z_{\Omega}, \mathcal{P}_{\Omega}\right)$-semistable, and let

$$
0=E_{0} \subset E_{1} \subset \ldots \subset E_{n-1} \subset E_{n}=E
$$

be the Jordan-Hölder filtration of $E$. Define

$$
\operatorname{Gr}(E)=\bigoplus_{i=1}^{n} E_{i} / E_{i-1}
$$

Two $\left(Z_{\Omega}, \mathcal{P}_{\Omega}\right)$-semistable objects $E_{1}, E_{2} \in \mathcal{P}_{\Omega}((0,1])$ are defined to be $S$ equivalent if $\operatorname{Gr}\left(E_{1}\right) \cong \operatorname{Gr}\left(E_{2}\right)$. Define $\overline{\mathfrak{M}}_{\Omega}(\mathfrak{t})$ to be the set of all $\left(Z_{\Omega}, \mathcal{P}_{\Omega}\right)$ semistable objects $E \in \mathcal{P}_{\Omega}((0,1])$ with $\mathfrak{t}(E)=\mathfrak{t}$ modulo $S$-equivalence.

(ii) For $m>0$, define the $S$-equivalence with respect to $\left(Z_{m}, \mathcal{P}_{m}\right)$ in a similar fashion as in (i), and define $\overline{\mathfrak{M}}_{\mathbf{u}_{m}}(\mathfrak{t})$ be the set of all $\left(Z_{m}, \mathcal{P}_{m}\right)$-semistable objects $E \in \mathcal{A}_{(\omega, \beta \omega)}^{\sharp}$ with $\mathfrak{t}(E)=\mathfrak{t}$ modulo $S$-equivalence.

(iii) Let $\overline{\mathfrak{M}}_{\omega}(\mathfrak{t})$ be the moduli space of sheaves $E \in \operatorname{Coh}(X)$ which are Simpsonsemistable with respect to $\omega$ and satisfy $\mathfrak{t}(E)=\mathfrak{t}$.

(iv) For $r>0$, define $\mathfrak{M}_{\omega}(\mathfrak{t})$ be the moduli space of locally free sheaves $E$ which are $\mu_{\omega}$-stable and satisfy $\mathfrak{t}(E)=\mathfrak{t}$. Define $\overline{\mathfrak{U}}_{\omega}(\mathfrak{t})$ to be the Uhlenbeck compactification space associated to $\omega$ and $\mathfrak{t}$.

Lemma 2.10. Let $\mathfrak{t}=(0,0, n)$ where $n \in \mathbb{Z}_{\geq 0}$. Then, all the spaces $\overline{\mathfrak{M}}_{\Omega}(\mathfrak{t})$, $\overline{\mathfrak{M}}_{\mathbf{u}_{m}}(\mathfrak{t})$ and $\overline{\mathfrak{M}}_{\omega}(\mathfrak{t})$ are identified, as sets, with the symmetric product $\operatorname{Sym}^{n}(X)$.

Proof. For $\overline{\mathfrak{M}}_{\omega}(\mathfrak{t})$, this follows from the fact that every 0-dimensional torsion sheaf is generated by the skyscraper sheaves $\mathcal{O}_{x}, x \in X$ via extensions. For $\overline{\mathfrak{M}}_{\Omega}(\mathfrak{t})$ 
(respectively, $\left.\overline{\mathfrak{M}}_{\mathbf{u}_{m}}(\mathfrak{t})\right)$, note that every skyscraper sheaf $\mathcal{O}_{x} \in \mathcal{P}_{\Omega}((0,1])$ has phase 1 and is $\left(Z_{\Omega}, \mathcal{P}_{\Omega}\right)$-stable by [Bay] (respectively, $\left(Z_{m}, \mathcal{P}_{m}\right)$-stable by [Bri2]).

Lemma 2.11. Let $\Omega=(\omega, \rho, p, U)$ be from Subsect. 2.2 with $U=e^{-K_{X} / 2}$. Let $\mathfrak{t}=\left(0, c_{1}, c_{2}\right)$ with $c_{1} \neq 0$. Then, $\overline{\mathfrak{M}}_{\Omega}(\mathfrak{t})$ is identified with $\overline{\mathfrak{M}}_{\omega}(\mathfrak{t})$.

Proof. We may let $c_{1}>0$. By the proof of Lemma 4.2 in [Bay], if $E \in \overline{\mathfrak{M}}_{\Omega}(\mathfrak{t})$ is $\left(Z_{\Omega}, \mathcal{P}_{\Omega}\right)$-semistable, then $E \in \overline{\mathfrak{M}}_{\omega}(\mathfrak{t})$ is Simpson-semistable with respect to $\omega$.

Conversely, let $E \in \overline{\mathfrak{M}}_{\omega}(\mathfrak{t})$ be Simpson-semistable with respect to $\omega$. Note that

$$
\begin{aligned}
\chi\left(E \otimes \mathcal{O}_{X}(m \omega)\right) & =\left(c_{1} \omega\right) m+\left(\mathrm{ch}_{2}-c_{1} \beta\right) \\
Z_{\Omega}(E)(m) & =i\left(c_{1} \omega\right) m-\left(\mathrm{ch}_{2}-c_{1} \beta\right)
\end{aligned}
$$

where $\beta=K_{X} / 2$ and $\mathrm{ch}_{2}=c_{1}^{2} / 2-c_{2}$. Let $A$ be any proper sub-object of $E$ in $\mathcal{P}_{\Omega}((0,1])=\mathcal{A}_{(\omega, \beta \omega)}^{\sharp}$, and let $B=E / A$. Then we have the exact sequence $0 \rightarrow A \rightarrow$ $E \rightarrow B \rightarrow 0$ in $\mathcal{P}_{\Omega}((0,1])$. Thus, $A$ is a sheaf in $\mathcal{T}_{(\omega, \beta \omega)}$ sitting in

$$
0 \rightarrow \mathcal{H}^{-1}(B) \rightarrow A \rightarrow E \rightarrow \mathcal{H}^{0}(B) \rightarrow 0 .
$$

If $\mathcal{H}^{-1}(B)=0$, then $A$ is a proper subsheaf of $E$. By (2.9),

$$
\left(\operatorname{ch}_{2}(A)-c_{1}(A) \beta\right) /\left(c_{1}(A) \omega\right) \leq\left(\operatorname{ch}_{2}-c_{1} \beta\right) /\left(c_{1} \omega\right)
$$

since $E$ is Simpson-semistable with respect to $\omega$. By $(2.10), \phi\left(Z_{\Omega}(E)(m)\right) \geq$ $\phi\left(Z_{\Omega}(A)(m)\right)$ for all $m>0$. Assume that $\mathcal{H}^{-1}(B) \neq 0$. By $(2.11)$, we obtain $\operatorname{rk}(A)=$ $\operatorname{rk}\left(\mathcal{H}^{-1}(B)\right)>0$. So we conclude from $(2.7)$ that $\phi\left(Z_{\Omega}(E)(m)\right)>\phi\left(Z_{\Omega}(A)(m)\right)$ for $m \gg 0$. Therefore, $E \in \overline{\mathfrak{M}}_{\Omega}(\mathfrak{t})$ is $\left(Z_{\Omega}, \mathcal{P}_{\Omega}\right)$-semistable.

Definition 2.12. (see [LQ]) Let $\mathbb{C}_{X} \subset \operatorname{Num}(X)_{\mathbb{R}}$ be the ample cone of the smooth projective surface $X$. Fix a numerical type $\mathfrak{t}=\left(r, c_{1}, c_{2}\right)$ on $X$.

(i) For a class $\xi \in \operatorname{Num}(X) \otimes \mathbb{R}$, we define

$$
W^{\xi}=\mathbb{C}_{X} \cap\left\{\alpha \in \operatorname{Num}(X)_{\mathbb{R}} \mid \alpha \cdot \xi=0\right\} .
$$

(ii) Let $\mathcal{W}(\mathfrak{t})$ be the set whose elements are of the form $W^{\xi}$, where $\xi$ is the numerical equivalence class $\left(r F-s c_{1}\right)$ for some divisor $F$ and some integer $s$ with $0<s<|r|$ satisfying the inequalities:

$$
-\frac{r^{2}}{4}\left(2 r c_{2}-(r-1) c_{1}^{2}\right) \leq \xi^{2}<0 .
$$

(iii) A wall of type $\mathfrak{t}$ is an element in $\mathcal{W}(\mathfrak{t})$, while a chamber of type $\mathfrak{t}$ is a connected component in the complement $\mathbb{C}_{X}-\mathcal{W}(\mathfrak{t})$.

It is well-known that the set $\mathcal{W}(\mathfrak{t})$ of walls of type $\mathfrak{t}$ is locally finite, i.e., given a compact subset $K$ of $\mathbb{C}_{X}$, there are only finitely many walls $W$ of type $\mathfrak{t}$ such that $W \cap K \neq \emptyset$. In addition, $\xi$ defines a wall of type $\mathfrak{t}$ if and only if it defines a wall of type $\left(\tilde{r}, \tilde{c}_{1}, \tilde{c}_{2}\right)$ where $\tilde{r}=-r$ and $1+\tilde{c}_{1}+\tilde{c}_{2}=\left(1+c_{1}+c_{2}\right)^{-1} \in A^{*}(X)$.

Fix $\mathfrak{t}=\left(r, c_{1}, c_{2}\right)$ with $r>0$. Then the Simpson-semistability is the same as the Gieseker-semistability. If $\omega_{1}$ and $\omega_{2}$ are contained in the same chamber of type $\mathfrak{t}$, then $\overline{\mathfrak{M}}_{\omega_{1}}(\mathfrak{t})=\overline{\mathfrak{M}}_{\omega_{2}}(\mathfrak{t})$. If $\omega$ is contained in a chamber of type $\mathfrak{t}$, then $E$ is $\mu_{\omega}$-stable whenever it is $\mu_{\omega}$-semistable and $\mathfrak{t}(E)=\left(r, c_{1}, c_{2}^{\prime}\right)$ with $c_{2}^{\prime} \leq c_{2}$. In this case,

$$
\overline{\mathfrak{U}}_{\omega}\left(r, c_{1}, c_{2}\right)=\bigoplus_{c_{2}^{\prime} \leq c_{2}} \mathfrak{M}_{\omega}\left(r, c_{1}, c_{2}^{\prime}\right) \times \operatorname{Sym}^{c_{2}-c_{2}^{\prime}}(X) .
$$

It was proved in $[\mathrm{Li} 1, \mathrm{Li} 2, \mathrm{Mor}]$ that $\overline{\mathfrak{U}}_{\omega}(\mathfrak{t})$ is a projective variety. 
3. Locally finiteness of mini-walls of a fixed type. In this section, we define and study the mini-walls of a fixed type $(\mathfrak{t}, \beta, \omega)$ for Bridgeland stability conditions. We will prove that the set of the mini-walls of a fixed type $(\mathfrak{t}, \beta, \omega)$ is locally finite.

Definition 3.1. Let $\sigma$ be a Bridgeland stability condition on $D^{b}(X)$. Let

$$
0=E_{0} \subset E_{1} \subset \ldots E_{n-1} \subset E_{n}=E
$$

be the HN-filtration of $E \in \mathcal{D}^{b}(X)$ with respect to $\sigma$. We define $E_{1}$ to be the leading $H N$-filtration component of $E$ with respect to $\sigma$.

Definition 3.2. Let $\mathbf{u}=e^{-(\beta+i \omega)}$ with $\omega$ being ample. Fix a numerical type $\mathfrak{t}=\left(r, c_{1}, c_{2}\right)$, and fix a subset $I$ of $(0,+\infty)$.

(i) A mini-wall of type $(\mathfrak{t}, \beta, \omega)$ in $I$ is a number $m_{0} \in I$ such that

$$
\phi\left(Z_{m_{0}}(A)\right)=\phi\left(Z_{m_{0}}(E)\right)
$$

where $E \in \overline{\mathfrak{M}}_{\mathbf{u}_{m_{1}}}(\mathfrak{t})$ for some $m_{1} \in I, E \notin \overline{\mathfrak{M}}_{\mathbf{u}_{m_{2}}}(\mathfrak{t})$ for some $m_{2} \in I$, and $A$ is the leading HN-filtration component of $E$ with respect to $\left(Z_{m_{2}}, \mathcal{P}_{m_{2}}\right)$.

(ii) A mini-chamber of type $(\mathfrak{t}, \beta, \omega)$ in $I$ is a connected component of

$$
I-\{m \mid m \text { is a mini-wall of type }(\mathfrak{t}, \beta, \omega) \text { in } I\} .
$$

REMARK 3.3. (i) Unlike Definition 2.12 (iii), our definitions of mini-walls and mini-chambers depends on subsets $I$ of $(0,+\infty)$. These dependences are consistent with the Proposition 9.3 in [Bri2] where when $X$ is a $K 3$ surface, walls and chambers are defined for compact subsets in the space of Bridgeland stability conditions.

(ii) Let $I_{1} \subset I_{2} \subset(0,+\infty)$. If $m$ is a mini-wall of type $(\mathfrak{t}, \beta, \omega)$ in $I_{1}$, then $m$ is a mini-wall of type $(\mathfrak{t}, \beta, \omega)$ in $I_{2}$. However, the converse may not be true.

(iii) Let $\mathfrak{t}=\left(0,0, c_{2}\right)$, and let $I \subset(0,+\infty)$ be connected. By Lemma 2.10, all the spaces $\overline{\mathfrak{M}}_{\mathbf{u}_{m}}(\mathfrak{t})$ with $m>0$ are identical. Hence no mini-walls of type $(\mathfrak{t}, \beta, \omega)$ in $I$ exist, and the only mini-chamber of type $(\mathfrak{t}, \beta, \omega)$ in $I$ is $I$ itself.

LEMmA 3.4. Let $\mathfrak{C}$ be a mini-chamber of type $(\mathfrak{t}, \beta, \omega)$ in I. If $m_{1}, m_{2} \in \mathfrak{C}$, then

$$
\overline{\mathfrak{M}}_{\mathbf{u}_{m_{1}}}(\mathfrak{t})=\overline{\mathfrak{M}}_{\mathbf{u}_{m_{2}}}(\mathfrak{t}) \text {. }
$$

Proof. By symmetry, it suffices to show that if $E \in \overline{\mathfrak{M}}_{\mathbf{u}_{m_{1}}}(\mathfrak{t})$, then $E \in \overline{\mathfrak{M}}_{\mathbf{u}_{m_{2}}}$ (t). Assume that $E \notin \overline{\mathfrak{M}}_{\mathbf{u}_{m_{2}}}(\mathfrak{t})$. Let $A$ be the leading HN-filtration component of $E$ with respect to $\left(Z_{m_{2}}, \mathcal{P}_{m_{2}}\right)$. Then $\phi\left(Z_{m_{2}}(A)\right)>\phi\left(Z_{m_{2}}(E)\right)$. Since $E \in \overline{\mathfrak{M}}_{\mathbf{u}_{m_{1}}}(\mathfrak{t})$, we have $\phi\left(Z_{m_{2}}(A)\right) \leq \phi\left(Z_{m_{2}}(E)\right)$. Since $\mathfrak{C}$ is connected, $\phi\left(Z_{m_{0}}(A)\right)=\phi\left(Z_{m_{0}}(E)\right)$ for some $m_{0} \in \mathfrak{C}$. Thus by definition, $m_{0}$ is a mini-wall of type $(\mathfrak{t}, \beta, \omega)$ in $I$. This is impossible since the mini-chamber $\mathfrak{C}$ can not contain any mini-wall.

Lemma 3.5. Let $\omega \in \operatorname{Num}(X)_{\mathbb{R}}$ be ample, and let $v \in \mathbb{R}$. Let $E \in \mathcal{A}_{(\omega, v)}^{\sharp}$.

(i) Let $f: \mathcal{H}^{0}(E) \rightarrow B$ be a surjection in $\operatorname{Coh}(X)$ with $\operatorname{ker}(f), B \in \mathcal{T}_{(\omega, v)}$. Then in $\mathcal{A}_{(\omega, v)}^{\sharp}$, there exists an exact sequence of the form

$$
0 \rightarrow A \rightarrow E \rightarrow B \rightarrow 0
$$


(ii) Let $g: A \rightarrow \mathcal{H}^{-1}(E)$ be an injection in $\operatorname{Coh}(X)$ with $A, \operatorname{coker}(g) \in \mathcal{F}_{(\omega, v)}$. Then in $\mathcal{A}_{(\omega, v)}^{\sharp}$, there exists an exact sequence of the form

$$
0 \rightarrow A[1] \rightarrow E \rightarrow B \rightarrow 0
$$

Proof. Since (ii) can be proved similarly, we will only prove (i) below. To prove (i), note from Definition 2.1 that $\mathcal{T}_{(\omega, v)} \subset \mathcal{A}_{(\omega, v)}^{\sharp}$. So $\operatorname{ker}(f), \mathcal{H}^{0}(E), B \in \mathcal{T}_{(\omega, v)} \subset \mathcal{A}_{(\omega, v)}^{\sharp}$. It follows that the exact sequence $0 \rightarrow \operatorname{ker}(f) \rightarrow \mathcal{H}^{0}(E) \rightarrow B \rightarrow 0$ in $\operatorname{Coh}(X)$ is an exact sequence in $\mathcal{A}_{(\omega, v)}^{\sharp}$. In particular, we have a surjection $\mathcal{H}^{0}(E) \rightarrow B$ in $\mathcal{A}_{(\omega, v)}^{\sharp}$. Composing with the surjection $E \rightarrow \mathcal{H}^{0}(E)$, we obtain a surjection $E \rightarrow B$ in $\mathcal{A}_{(\omega, v)}^{\sharp}$. Letting $A$ be the kernel of $E \rightarrow B$ in $\mathcal{A}_{(\omega, v)}^{\sharp}$, we obtain (3.1).

Lemma 3.6. Let $\omega \in \operatorname{Num}(X)_{\mathbb{R}}$ be ample, and let $v \in \mathbb{R}$.

(i) If $E \in \mathcal{A}_{(\omega, v)}^{\sharp}$, then $c_{1}(E) \omega \geq \operatorname{rk}(E) v$.

(ii) Fix a numerical type $\mathfrak{t}=\left(r, c_{1}, c_{2}\right)$. Let $\left\{E_{n}\right\}_{n=1}^{+\infty}$ be a sequence of objects in $\mathcal{A}_{(\omega, v)}^{\sharp}$. Assume that for each $n$, there exists an exact sequence

$$
0 \rightarrow A_{n} \rightarrow E_{n} \rightarrow B_{n} \rightarrow 0
$$

in $\mathcal{A}_{(\omega, v)}^{\sharp}$. Then, $\lim _{n \rightarrow+\infty} c_{1}\left(A_{n}\right) \omega / \operatorname{rk}\left(A_{n}\right)=v$ if $\lim _{n \rightarrow+\infty} \operatorname{rk}\left(A_{n}\right)=\infty$, and $\lim _{n \rightarrow+\infty} c_{1}\left(B_{n}\right) \omega / \operatorname{rk}\left(B_{n}\right)=v$ if $\lim _{n \rightarrow+\infty} \operatorname{rk}\left(B_{n}\right)=\infty$.

Proof. (i) Note that every $E \in \mathcal{A}_{(\omega, v)}^{\sharp}$ sits in the exact sequence

$$
0 \rightarrow \mathcal{H}^{-1}(E)[1] \rightarrow E \rightarrow \mathcal{H}^{0}(E) \rightarrow 0
$$

in $\mathcal{A}_{(\omega, v)}^{\sharp}$. Since $\mathcal{H}^{-1}(E) \in \mathcal{F}_{(\omega, v)}$ and $\mathcal{H}^{0}(E) \in \mathcal{T}_{(\omega, v)}$, we have $c_{1}\left(\mathcal{H}^{-1}(E)\right) \omega \leq$ $\operatorname{rk}\left(\mathcal{H}^{-1}(E)\right) v$ and $c_{1}\left(\mathcal{H}^{0}(E)\right) \omega \geq \operatorname{rk}\left(\mathcal{H}^{0}(E)\right) v$. So $c_{1}(E) \omega \geq \operatorname{rk}(E) v$.

(ii) Since the second statement can be proved similarly, we will only prove the first statement. Assume that $\lim _{n \rightarrow+\infty} \operatorname{rk}\left(A_{n}\right)=\infty$. By (i), we have $c_{1}\left(A_{n}\right) \omega \geq \operatorname{rk}\left(A_{n}\right) v$ and $c_{1}\left(B_{n}\right) \omega \geq \operatorname{rk}\left(B_{n}\right) v$. Therefore, we conclude that

$$
\operatorname{rk}\left(A_{n}\right) v \leq c_{1}\left(A_{n}\right) \omega=c_{1} \omega-c_{1}\left(B_{n}\right) \omega \leq c_{1} \omega-\operatorname{rk}\left(B_{n}\right) v=\left(c_{1} \omega-r v\right)+\operatorname{rk}\left(A_{n}\right) v .
$$

Since $\lim _{n \rightarrow+\infty} \operatorname{rk}\left(A_{n}\right)=\infty$, it follows that $\lim _{n \rightarrow+\infty} c_{1}\left(A_{n}\right) \omega / \operatorname{rk}\left(A_{n}\right)=v$.

Lemma 3.7. Let $\alpha, \omega \in \operatorname{Num}(X)_{\mathbb{R}}$ with $\omega$ being ample.

(i) If $c \leq \alpha \cdot \omega \leq d$, then $\alpha^{2} \leq \max \left\{c^{2}, d^{2}\right\} / \omega^{2}$.

(ii) Let $B$ be a torsion free $\mu_{\omega}$-semistable sheaf. If $c \leq \mu_{\omega}(B) \leq d$, then $c(B) / \operatorname{rk}(B)$ is bounded from below by a constant depending only on $c, d, \omega, \beta$.

Proof. (i) Write $\alpha=a \omega+\rho$ with $\rho \cdot \omega=0$. Then, $a=(\alpha \cdot \omega) / \omega^{2}$. By the Hodge Index Theorem, we have $\rho^{2} \leq 0$. It follows that

$$
\alpha^{2}=a^{2} \omega^{2}+\rho^{2} \leq a^{2} \omega^{2}=(\alpha \cdot \omega)^{2} / \omega^{2} \leq \max \left\{c^{2}, d^{2}\right\} / \omega^{2} .
$$

(ii) Since $c \leq \mu_{\omega}(B) \leq d$, we obtain the inequalities

$$
c-\beta \cdot \omega \leq\left(\frac{c_{1}(B)}{\operatorname{rk}(B)}-\beta\right) \cdot \omega \leq d-\beta \cdot \omega .
$$


By the Bogomolov Inequality, $\operatorname{ch}_{2}(B) \leq c_{1}(B)^{2} /(2 \operatorname{rk}(B))$. By (2.6),

$$
\frac{c(B)}{\operatorname{rk}(B)}=-\frac{\operatorname{ch}_{2}(B)}{\operatorname{rk}(B)}+\frac{c_{1}(B)}{\operatorname{rk}(B)} \cdot \beta-\frac{\beta^{2}}{2} \geq-\frac{1}{2}\left(\frac{c_{1}(B)}{\operatorname{rk}(B)}-\beta\right)^{2} .
$$

Now our conclusion about $c(B) / \operatorname{rk}(B)$ follows immediately from (i).

Proposition 3.8. Let $\mathbf{u}=e^{-(\beta+i \omega)}$ with $\omega$ being ample and $\beta, \omega \in \operatorname{Num}(X)_{\mathbb{R}}$. Fix a numerical type $\mathfrak{t}=\left(r, c_{1}, c_{2}\right)$ and an interval $I=[a,+\infty)$ with $a>0$. Then there exists a positive number $N$ depending only on $\mathfrak{t}, \beta, \omega$ and I such that $\operatorname{rk}\left(\mathcal{H}^{0}(E)\right)$, $\operatorname{rk}\left(\mathcal{H}^{-1}(E)\right) \leq N$ whenever $E \in \overline{\mathfrak{M}}_{\mathbf{u}_{m}}(\mathfrak{t})$ for some $m \in I$.

Proof. Since $\operatorname{rk}\left(\mathcal{H}^{-1}(E)\right)=\operatorname{rk}\left(\mathcal{H}^{0}(E)\right)-r$, it suffices to prove the lemma for $\operatorname{rk}\left(\mathcal{H}^{0}(E)\right)$. Assume that such an $N$ does not exist for $\operatorname{rk}\left(\mathcal{H}^{0}(E)\right)$. Then there exists a sequence of objects $E_{n} \in \overline{\mathfrak{M}}_{\mathbf{u}_{m_{n}}}(\mathfrak{t}), n=1,2, \ldots$, with $m_{n} \in I$ for all $n$ and $\lim _{n \rightarrow+\infty} \operatorname{rk}\left(\mathcal{H}^{0}\left(E_{n}\right)\right)=+\infty$. Replacing by a subsequence if necessary, we may further assume that $\lim _{n \rightarrow+\infty} m_{n}=m_{0}$ (possibly $+\infty$ ).

To draw a contradiction, let $A_{n, 0}=\mathcal{H}^{0}\left(E_{n}\right)$ and $A_{n, 1}=\mathcal{H}^{-1}\left(E_{n}\right)$. Then we have the exact sequence $0 \rightarrow A_{n, 1}[-1] \rightarrow E_{n} \rightarrow A_{n, 0} \rightarrow 0$ in $\mathcal{A}_{(\omega, \beta \omega)}^{\sharp}$. By Lemma 3.6 (ii), $\lim _{n \rightarrow+\infty} \mu_{\omega}\left(A_{n, 0}\right)=\beta \omega$. Let $F_{n}=A_{n, 0} / \operatorname{Tor}\left(A_{n, 0}\right)$. Let $F_{n}^{(1)}, \ldots, F_{n}^{\left(\ell_{n}\right)}$ be the usual HN-filtration quotients of $F_{n}$ with respect to $\mu_{\omega}$, i.e., $F_{n}^{(i)}=\mathcal{F}_{i}\left(F_{n}\right) / \mathcal{F}_{i-1}\left(F_{n}\right)$ if the usual HN-filtration of $F_{n}$ with respect to $\mu_{\omega}$ is given by

$$
0=\mathcal{F}_{0}\left(F_{n}\right) \subset \mathcal{F}_{1}\left(F_{n}\right) \subset \mathcal{F}_{2}\left(F_{n}\right) \subset \cdots \subset \mathcal{F}_{\ell_{n}}\left(F_{n}\right)=F_{n} .
$$

Then $F_{n}^{(1)}, \ldots, F_{n}^{\left(\ell_{n}\right)}$ are torsion free and $\mu_{\omega}$-semistable, and

$$
\mu_{\omega}\left(F_{n}^{(1)}\right)>\ldots>\mu_{\omega}\left(F_{n}^{\left(\ell_{n}\right)}\right) .
$$

Moreover, $\beta \omega<\mu_{\omega}\left(F_{n}^{\left(\ell_{n}\right)}\right) \leq \mu_{\omega}\left(F_{n}\right) \leq \mu_{\omega}\left(A_{n, 0}\right)$. Thus $\lim _{n \rightarrow+\infty} \mu_{\omega}\left(F_{n}^{\left(\ell_{n}\right)}\right)=\beta \omega$. In particular, for $n \gg 0$, we have $\beta \omega<\mu_{\omega}\left(F_{n}^{\left(\ell_{n}\right)}\right) \leq \beta \omega+\epsilon_{n}$ where $\left\{\epsilon_{n}\right\}_{n \gg 0}$ is a sequence of positive numbers with $\lim _{n \rightarrow+\infty} \epsilon_{n}=0$. By the proof of Lemma 3.7,

$$
\frac{c\left(F_{n}^{\left(\ell_{n}\right)}\right)}{\operatorname{rk}\left(F_{n}^{\left(\ell_{n}\right)}\right)} \geq-\frac{1}{2}\left(\frac{c_{1}\left(F_{n}^{\left(\ell_{n}\right)}\right)}{\operatorname{rk}\left(F_{n}^{\left(\ell_{n}\right)}\right)}-\beta\right)^{2} \geq-\frac{\epsilon_{n}^{2}}{2 \omega^{2}} .
$$

On the other hand, by Lemma 3.5 (i), there exists a quotient $E_{n} \rightarrow F_{n}^{\left(\ell_{n}\right)} \rightarrow 0$ in $\mathcal{A}_{(\omega, \beta \omega)}^{\sharp}$. Since $E_{n} \in \overline{\mathfrak{M}}_{\mathbf{u}_{m_{n}}}(\mathfrak{t})$, we see from (2.7) that

$$
\begin{aligned}
& \frac{\omega^{2} m_{n}^{2}}{2}\left(r c_{1}\left(F_{n}^{\left(\ell_{n}\right)}\right) \omega-\operatorname{rk}\left(F_{n}^{\left(\ell_{n}\right)}\right) c_{1} \omega\right) \\
\geq & c\left(F_{n}^{\left(\ell_{n}\right)}\right)\left(c_{1} \omega-r \beta \omega\right)-c(\mathfrak{t}, \beta)\left(c_{1}\left(F_{n}^{\left(\ell_{n}\right)}\right) \omega-\operatorname{rk}\left(F_{n}^{\left(\ell_{n}\right)}\right) \beta \omega\right)
\end{aligned}
$$

where $c(\mathfrak{t}, \beta)=c\left(E_{n}\right)$ depends only on $\mathfrak{t}$ and $\beta$. By Lemma 3.6 (i), $c_{1} \omega-r \beta \omega \geq 0$. If $c_{1} \omega-r \beta \omega=0$, then the phase of $E_{n}$ with respect to every central charge $Z_{\mathbf{u}_{m}}, m>0$ is equal to 1. So $E_{n} \in \overline{\mathfrak{M}}_{\mathbf{u}_{m}}(\mathfrak{t})$ for all $n, m>0$. In view of Lemma 2.6 and Lemma 2.4, this contradicts to $\lim _{n \rightarrow+\infty} \operatorname{rk}\left(\mathcal{H}^{0}\left(E_{n}\right)\right)=+\infty$. Thus $c_{1} \omega-r \beta \omega>0$ and

$$
\frac{c\left(F_{n}^{\left(\ell_{n}\right)}\right)}{\operatorname{rk}\left(F_{n}^{\left(\ell_{n}\right)}\right)} \leq \frac{\omega^{2} m_{n}^{2}}{2} \cdot \frac{r \mu_{\omega}\left(F_{n}^{\left(\ell_{n}\right)}\right)-c_{1} \omega}{c_{1} \omega-r \beta \omega}+c(\mathfrak{t}, \beta) \cdot \frac{\mu_{\omega}\left(F_{n}^{\left(\ell_{n}\right)}\right)-\beta \omega}{c_{1} \omega-r \beta \omega} .
$$


Combining this with (3.4), we conclude that

$$
\frac{\omega^{2} m_{n}^{2}}{2} \cdot \frac{r \mu_{\omega}\left(F_{n}^{\left(\ell_{n}\right)}\right)-c_{1} \omega}{c_{1} \omega-r \beta \omega}+c(\mathfrak{t}, \beta) \cdot \frac{\mu_{\omega}\left(F_{n}^{\left(\ell_{n}\right)}\right)-\beta \omega}{c_{1} \omega-r \beta \omega} \geq-\frac{\epsilon_{n}^{2}}{2 \omega^{2}} .
$$

Recall that $\lim _{n \rightarrow+\infty} \mu_{\omega}\left(F_{n}^{\left(\ell_{n}\right)}\right)=\beta \omega$ and $\lim _{n \rightarrow+\infty} m_{n}=m_{0} \geq a>0$. Letting $n \rightarrow+\infty$, we see that $-\omega^{2} m_{0}^{2} / 2 \geq 0$ which is impossible.

Lemma 3.9. Let $\mathbf{u}=e^{-(\beta+i \omega)}$ with $\omega$ being ample and $\beta, \omega \in \operatorname{Num}(X)_{\mathbb{R}}$. Fix a numerical type $\mathfrak{t}=\left(r, c_{1}, c_{2}\right)$ and an interval $I=[a,+\infty)$ with $a>0$. Then there exists $N$ depending only on $\mathfrak{t}, \beta, \omega$ and $I$ such that $|\operatorname{rk}(A)| \leq N$ whenever

- $A$ is $\left(Z_{m_{2}}, \mathcal{P}_{m_{2}}\right)$-semistable for some $m_{2} \in I$;

- $A$ is a proper sub-object of certain object $E \in \mathcal{A}_{(\omega, \beta \omega)}^{\sharp}$ with $\mathfrak{t}(E)=\mathfrak{t}$;

- $A$ destablizes $E$ with respect to $\left(Z_{m_{2}}, \mathcal{P}_{m_{2}}\right)$;

- $\phi\left(Z_{m_{0}}(A)\right)=\phi\left(Z_{m_{0}}(E)\right)$ for some $m_{0} \in I$.

Proof. Assume that our statement is not true. Then there exists a sequence of sub-objects $A_{n} \subset E_{n}(n=1,2, \ldots)$ in $\mathcal{A}_{(\omega, \beta \omega)}^{\sharp}$ such that $\lim _{n \rightarrow+\infty} \operatorname{rk}\left(A_{n}\right)= \pm \infty$, $\mathfrak{t}\left(E_{n}\right)=\mathfrak{t}, A_{n}$ is $\left(Z_{m_{2, n}}, \mathcal{P}_{m_{2, n}}\right)$-semistable for some $m_{2, n} \in I, A_{n}$ destablizes $E_{n}$ with respect to $\left(Z_{m_{2, n}}, \mathcal{P}_{m_{2, n}}\right)$, and $\phi\left(Z_{m_{0, n}}\left(A_{n}\right)\right)=\phi\left(Z_{m_{0, n}}\left(E_{n}\right)\right)$ for some $m_{0, n} \in I$. We may assume that $\lim _{n \rightarrow+\infty} m_{i, n}=m_{i}$ (possibly $+\infty$ ) for $i=0,2$. Define

$$
d\left(\mathfrak{t}, \beta, \omega, A_{n}\right)=c\left(A_{n}\right)\left(c_{1} \omega-r \beta \omega\right)-c(\mathfrak{t}, \beta)\left(c_{1}\left(A_{n}\right) \omega-\operatorname{rk}\left(A_{n}\right) \beta \omega\right)
$$

where $c(\mathfrak{t}, \beta)=c\left(E_{n}\right)$ depends only on $\mathfrak{t}$ and $\beta$. Since $\phi\left(Z_{m_{0, n}}\left(A_{n}\right)\right)=\phi\left(Z_{m_{0, n}}\left(E_{n}\right)\right)$ and $\phi\left(Z_{m_{2, n}}\left(A_{n}\right)\right)>\phi\left(Z_{m_{2, n}}\left(E_{n}\right)\right)$, we see from (2.7) that

$$
\begin{aligned}
& \frac{\omega^{2} m_{0, n}^{2}}{2}\left(r c_{1}\left(A_{n}\right) \omega-\operatorname{rk}\left(A_{n}\right) c_{1} \omega\right)=d\left(\mathfrak{t}, \beta, \omega, A_{n}\right), \\
& \frac{\omega^{2} m_{2, n}^{2}}{2}\left(r c_{1}\left(A_{n}\right) \omega-\operatorname{rk}\left(A_{n}\right) c_{1} \omega\right)>d\left(\mathfrak{t}, \beta, \omega, A_{n}\right) .
\end{aligned}
$$

Since $E_{n}$ is not $\left(Z_{m_{2, n}}, \mathcal{P}_{m_{2, n}}\right)$-semistable, we must have $\phi\left(Z_{m_{2, n}}\left(E_{n}\right)\right)<1$. So $c_{1} \omega-r \beta \omega>0$. This in turn implies that $\phi\left(Z_{m_{0, n}}\left(A_{n}\right)\right)=\phi\left(Z_{m_{0, n}}\left(E_{n}\right)\right)<1$. Therefore, $c_{1}\left(A_{n}\right) \omega-\operatorname{rk}\left(A_{n}\right) \beta \omega>0$. In summary, we obtain

$$
c_{1} \omega-r \beta \omega>0, c_{1}\left(A_{n}\right) \omega-\operatorname{rk}\left(A_{n}\right) \beta \omega>0 .
$$

By Lemma 3.6 (ii), $\lim _{n \rightarrow+\infty} \mu_{\omega}\left(A_{n}\right)=\beta \omega$. Dividing both sides of (3.6) by $\operatorname{rk}\left(A_{n}\right)\left(c_{1} \omega-r \beta \omega\right)$ and using (3.5), we conclude that

$$
\frac{c\left(A_{n}\right)}{\operatorname{rk}\left(A_{n}\right)}=\frac{\omega^{2} m_{0, n}^{2}}{2} \cdot \frac{r \mu_{\omega}\left(A_{n}\right)-c_{1} \omega}{c_{1} \omega-r \beta \omega}+c(\mathfrak{t}, \beta) \cdot \frac{\mu_{\omega}\left(A_{n}\right)-\beta \omega}{c_{1} \omega-r \beta \omega} .
$$

Let $A_{n, 0}=\mathcal{H}^{0}\left(A_{n}\right), A_{n, 1}=\mathcal{H}^{-1}\left(A_{n}\right)$ and $F_{n}=A_{n, 0} / \operatorname{Tor}\left(A_{n, 0}\right)$.

Case 1: $\lim _{n \rightarrow+\infty} \operatorname{rk}\left(A_{n}\right)=+\infty$. Then $\lim _{n \rightarrow+\infty} \operatorname{rk}\left(A_{n, 0}\right)=+\infty$. Since $A_{n, 1} \in$ $\mathcal{F}_{(\omega, \beta \omega)}$, we get $c_{1}\left(A_{n, 1}\right) \omega \leq \operatorname{rk}\left(A_{n, 1}\right) \beta \omega$. Since $F_{n} \in \mathcal{T}_{(\omega, \beta \omega)}$,

$$
\begin{aligned}
\beta \omega & <\mu_{\omega}\left(F_{n}\right) \leq \mu_{\omega}\left(A_{n, 0}\right)=\frac{c_{1}\left(A_{n}\right) \omega+c_{1}\left(A_{n, 1}\right) \omega}{\operatorname{rk}\left(A_{n, 0}\right)} \\
& \leq \frac{c_{1}\left(A_{n}\right) \omega+\operatorname{rk}\left(A_{n, 1}\right) \beta \omega}{\operatorname{rk}\left(A_{n, 0}\right)}=\frac{c_{1}\left(A_{n}\right) \omega+\left[\operatorname{rk}\left(A_{n, 0}\right)-\operatorname{rk}\left(A_{n}\right)\right] \beta \omega}{\operatorname{rk}\left(A_{n, 0}\right)} \\
& =\beta \omega+\frac{\operatorname{rk}\left(A_{n}\right)}{\operatorname{rk}\left(A_{n, 0}\right)} \cdot\left(\mu_{\omega}\left(A_{n}\right)-\beta \omega\right) \leq \beta \omega+\left(\mu_{\omega}\left(A_{n}\right)-\beta \omega\right)=\mu_{\omega}\left(A_{n}\right) .
\end{aligned}
$$


So $\lim _{n \rightarrow+\infty} \mu_{\omega}\left(F_{n}\right)=\beta \omega$. Let $F_{n}^{(1)}, \ldots, F_{n}^{\left(\ell_{n}\right)}$ be the usual HN-filtration quotients of $F_{n}$ with respect to $\mu_{\omega}$ such that $\mu_{\omega}\left(F_{n}^{(1)}\right)>\ldots>\mu_{\omega}\left(F_{n}^{\left(\ell_{n}\right)}\right)$. Then $F_{n}^{(1)}, \ldots, F_{n}^{\left(\ell_{n}\right)}$ are torsion free and $\mu_{\omega}$-semistable. Moreover, $\beta \omega<\mu_{\omega}\left(F_{n}^{\left(\ell_{n}\right)}\right) \leq \mu_{\omega}\left(F_{n}\right) \leq \mu_{\omega}\left(A_{n}\right)$. Thus $\lim _{n \rightarrow+\infty} \mu_{\omega}\left(F_{n}^{\left(\ell_{n}\right)}\right)=\beta \omega$, and $\beta \omega<\mu_{\omega}\left(F_{n}^{\left(\ell_{n}\right)}\right) \leq \beta \omega+\epsilon_{n}$ for $n \gg 0$ where $\left\{\epsilon_{n}\right\}_{n \gg 0}$ is a sequence of positive numbers with $\lim _{n \rightarrow+\infty} \epsilon_{n}=0$. As in (3.4),

$$
\frac{c\left(F_{n}^{\left(\ell_{n}\right)}\right)}{\operatorname{rk}\left(F_{n}^{\left(\ell_{n}\right)}\right)} \geq-\frac{\epsilon_{n}^{2}}{2 \omega^{2}}
$$

On the other hand, by Lemma 3.5 (i), there exists a quotient $A_{n} \rightarrow F_{n}^{\left(\ell_{n}\right)} \rightarrow 0$ in $\mathcal{A}_{(\omega, \beta \omega)}^{\sharp}$. Since $A_{n}$ is $\left(Z_{m_{2, n}}, \mathcal{P}_{m_{2, n}}\right)$-semistable, we have

$$
\begin{aligned}
& \frac{\omega^{2} m_{2, n}^{2}}{2}\left(\operatorname{rk}\left(A_{n}\right) c_{1}\left(F_{n}^{\left(\ell_{n}\right)}\right) \omega-\operatorname{rk}\left(F_{n}^{\left(\ell_{n}\right)}\right) c_{1}\left(A_{n}\right) \omega\right) \\
\geq & c\left(F_{n}^{\left(\ell_{n}\right)}\right)\left(c_{1}\left(A_{n}\right) \omega-\operatorname{rk}\left(A_{n}\right) \beta \omega\right)-c\left(A_{n}\right)\left(c_{1}\left(F_{n}^{\left(\ell_{n}\right)}\right) \omega-\operatorname{rk}\left(F_{n}^{\left(\ell_{n}\right)}\right) \beta \omega\right) .
\end{aligned}
$$

by (2.7). Since $c_{1}\left(A_{n}\right) \omega-\operatorname{rk}\left(A_{n}\right) \beta \omega>0$, we obtain

$$
\begin{aligned}
\frac{c\left(F_{n}^{\left(\ell_{n}\right)}\right)}{\operatorname{rk}\left(F_{n}^{\left(\ell_{n}\right)}\right)} \leq & \frac{c\left(A_{n}\right)}{\operatorname{rk}\left(A_{n}\right)} \cdot \frac{\mu_{\omega}\left(F_{n}^{\left(\ell_{n}\right)}\right)-\beta \omega}{\mu_{\omega}\left(A_{n}\right)-\beta \omega}+\frac{\omega^{2} m_{2, n}^{2}}{2} \cdot \frac{\mu_{\omega}\left(F_{n}^{\left(\ell_{n}\right)}\right)-\mu_{\omega}\left(A_{n}\right)}{\mu_{\omega}\left(A_{n}\right)-\beta \omega} \\
= & \frac{\omega^{2} m_{0, n}^{2}}{2} \cdot \frac{r \mu_{\omega}\left(A_{n}\right)-c_{1} \omega}{c_{1} \omega-r \beta \omega} \cdot \frac{\mu_{\omega}\left(F_{n}^{\left(\ell_{n}\right)}\right)-\beta \omega}{\mu_{\omega}\left(A_{n}\right)-\beta \omega} \\
& +c(\mathfrak{t}, \beta) \cdot \frac{\mu_{\omega}\left(F_{n}^{\left(\ell_{n}\right)}\right)-\beta \omega}{c_{1} \omega-r \beta \omega}+\frac{\omega^{2} m_{2, n}^{2}}{2} \cdot \frac{\mu_{\omega}\left(F_{n}^{\left(\ell_{n}\right)}\right)-\mu_{\omega}\left(A_{n}\right)}{\mu_{\omega}\left(A_{n}\right)-\beta \omega}
\end{aligned}
$$

where we have used (3.9) in the second step. Combining with (3.10), we get

$$
\begin{aligned}
-\frac{\epsilon_{n}^{2}}{2 \omega^{2}} \leq & \frac{\omega^{2} m_{0, n}^{2}}{2} \cdot \frac{r \mu_{\omega}\left(A_{n}\right)-c_{1} \omega}{c_{1} \omega-r \beta \omega} \cdot \frac{\mu_{\omega}\left(F_{n}^{\left(\ell_{n}\right)}\right)-\beta \omega}{\mu_{\omega}\left(A_{n}\right)-\beta \omega} \\
& +\frac{\omega^{2} m_{2, n}^{2}}{2} \cdot \frac{\mu_{\omega}\left(F_{n}^{\left(\ell_{n}\right)}\right)-\mu_{\omega}\left(A_{n}\right)}{\mu_{\omega}\left(A_{n}\right)-\beta \omega}+c(\mathfrak{t}, \beta) \cdot \frac{\mu_{\omega}\left(F_{n}^{\left(\ell_{n}\right)}\right)-\beta \omega}{c_{1} \omega-r \beta \omega} .
\end{aligned}
$$

Note that we may assume either $m_{0, n}<m_{2, n}$ for all $n$ or $m_{0, n}>m_{2, n}$ for all $n$. If $m_{0, n}<m_{2, n}$ for all $n$, then since $\mu_{\omega}\left(F_{n}^{\left(\ell_{n}\right)}\right)-\mu_{\omega}\left(A_{n}\right) \leq 0$, we see from (3.11) that

$$
\begin{aligned}
-\frac{\epsilon_{n}^{2}}{2 \omega^{2}} \leq & \frac{\omega^{2} m_{0, n}^{2}}{2} \cdot \frac{r \mu_{\omega}\left(A_{n}\right)-c_{1} \omega}{c_{1} \omega-r \beta \omega} \cdot \frac{\mu_{\omega}\left(F_{n}^{\left(\ell_{n}\right)}\right)-\beta \omega}{\mu_{\omega}\left(A_{n}\right)-\beta \omega} \\
& +\frac{\omega^{2} m_{0, n}^{2}}{2} \cdot \frac{\mu_{\omega}\left(F_{n}^{\left(\ell_{n}\right)}\right)-\mu_{\omega}\left(A_{n}\right)}{\mu_{\omega}\left(A_{n}\right)-\beta \omega}+c(\mathfrak{t}, \beta) \cdot \frac{\mu_{\omega}\left(F_{n}^{\left(\ell_{n}\right)}\right)-\beta \omega}{c_{1} \omega-r \beta \omega} \\
= & \frac{\omega^{2} m_{0, n}^{2}}{2} \cdot \frac{r \mu_{\omega}\left(F_{n}^{\left(\ell_{n}\right)}\right)-c_{1} \omega}{c_{1} \omega-r \beta \omega}+c(\mathfrak{t}, \beta) \cdot \frac{\mu_{\omega}\left(F_{n}^{\left(\ell_{n}\right)}\right)-\beta \omega}{c_{1} \omega-r \beta \omega} .
\end{aligned}
$$

Letting $n \rightarrow+\infty$, we obtain $0 \leq-\omega^{2} m_{0}^{2} / 2$ which is impossible since $m_{0} \geq a>0$. Similarly, if $m_{0, n}>m_{2, n}$ for all $n$, then $r \mu_{\omega}\left(A_{n}\right)-c_{1} \omega<0$ by (3.6) and (3.7). 
Therefore, we conclude from (3.11) again that

$$
\begin{aligned}
-\frac{\epsilon_{n}^{2}}{2 \omega^{2}}< & \frac{\omega^{2} m_{2, n}^{2}}{2} \cdot \frac{r \mu_{\omega}\left(A_{n}\right)-c_{1} \omega}{c_{1} \omega-r \beta \omega} \cdot \frac{\mu_{\omega}\left(F_{n}^{\left(\ell_{n}\right)}\right)-\beta \omega}{\mu_{\omega}\left(A_{n}\right)-\beta \omega} \\
& +\frac{\omega^{2} m_{2, n}^{2}}{2} \cdot \frac{\mu_{\omega}\left(F_{n}^{\left(\ell_{n}\right)}\right)-\mu_{\omega}\left(A_{n}\right)}{\mu_{\omega}\left(A_{n}\right)-\beta \omega}+c(\mathfrak{t}, \beta) \cdot \frac{\mu_{\omega}\left(F_{n}^{\left(\ell_{n}\right)}\right)-\beta \omega}{c_{1} \omega-r \beta \omega} \\
= & \frac{\omega^{2} m_{2, n}^{2}}{2} \cdot \frac{r \mu_{\omega}\left(F_{n}^{\left(\ell_{n}\right)}\right)-c_{1} \omega}{c_{1} \omega-r \beta \omega}+c(\mathfrak{t}, \beta) \cdot \frac{\mu_{\omega}\left(F_{n}^{\left(\ell_{n}\right)}\right)-\beta \omega}{c_{1} \omega-r \beta \omega} .
\end{aligned}
$$

Letting $n \rightarrow+\infty$, we obtain $0 \leq-\omega^{2} m_{2}^{2} / 2$ which is impossible since $m_{2} \geq a>0$.

Case 2: $\lim _{n \rightarrow+\infty} \operatorname{rk}\left(A_{n}\right)=-\infty$. Then $\lim _{n \rightarrow+\infty} \operatorname{rk}\left(A_{n, 1}\right)=+\infty$ and

$$
\begin{aligned}
\beta \omega & \geq \mu_{\omega}\left(A_{n, 1}\right)=\frac{c_{1}\left(A_{n, 0}\right) \omega-c_{1}\left(A_{n}\right) \omega}{\operatorname{rk}\left(A_{n, 1}\right)} \geq \frac{\operatorname{rk}\left(A_{n, 0}\right) \beta \omega-c_{1}\left(A_{n}\right) \omega}{\operatorname{rk}\left(A_{n, 1}\right)} \\
& =\frac{\left[\operatorname{rk}\left(A_{n, 1}\right)+\operatorname{rk}\left(A_{n}\right)\right] \beta \omega-c_{1}\left(A_{n}\right) \omega}{\operatorname{rk}\left(A_{n, 1}\right)}=\beta \omega-\frac{\operatorname{rk}\left(A_{n}\right)}{\operatorname{rk}\left(A_{n, 1}\right)} \cdot\left(\mu_{\omega}\left(A_{n}\right)-\beta \omega\right) \\
& \geq \beta \omega+\left(\mu_{\omega}\left(A_{n}\right)-\beta \omega\right)=\mu_{\omega}\left(A_{n}\right) .
\end{aligned}
$$

So $\lim _{n \rightarrow+\infty} \mu_{\omega}\left(A_{n, 1}\right)=\beta \omega$. Let $G_{n}^{(1)}, \ldots, G_{n}^{\left(k_{n}\right)}$ be the usual HN-filtration quotients of $A_{n, 1}$ with respect to $\mu_{\omega}$ such that $\mu_{\omega}\left(G_{n}^{(1)}\right)>\ldots>\mu_{\omega}\left(G_{n}^{\left(k_{n}\right)}\right)$. Then $G_{n}^{(1)}$ is torsion free and $\mu_{\omega}$-semistable. Moreover, $\beta \omega \geq \mu_{\omega}\left(G_{n}^{(1)}\right) \geq \mu_{\omega}\left(A_{n, 1}\right) \geq \mu_{\omega}\left(A_{n}\right)$. Thus $\lim _{n \rightarrow+\infty} \mu_{\omega}\left(G_{n}^{(1)}\right)=\beta \omega$, and $\beta \omega \geq \mu_{\omega}\left(G_{n}^{(1)}\right) \geq \beta \omega-\epsilon_{n}$ for $n \gg 0$ where $\left\{\epsilon_{n}\right\}_{n \gg 0}$ is a sequence of positive numbers with $\lim _{n \rightarrow+\infty} \epsilon_{n}=0$. As in (3.4),

$$
\frac{c\left(G_{n}^{(1)}\right)}{\operatorname{rk}\left(G_{n}^{(1)}\right)} \geq-\frac{\epsilon_{n}^{2}}{2 \omega^{2}} .
$$

On the other hand, by Lemma 3.5 (ii), there exists an injection $0 \rightarrow G_{n}^{(1)}[1] \rightarrow A_{n}$ in $\mathcal{A}_{(\omega, \beta \omega)}^{\sharp}$. Since $A_{n}$ is $\left(Z_{m_{2, n}}, \mathcal{P}_{m_{2, n}}\right)$-semistable, we have

$$
\begin{aligned}
& -\frac{\omega^{2} m_{2, n}^{2}}{2}\left(\operatorname{rk}\left(A_{n}\right) c_{1}\left(G_{n}^{(1)}\right) \omega-\operatorname{rk}\left(G_{n}^{(1)}\right) c_{1}\left(A_{n}\right) \omega\right) \\
\leq & -c\left(G_{n}^{(1)}\right)\left(c_{1}\left(A_{n}\right) \omega-\operatorname{rk}\left(A_{n}\right) \beta \omega\right)+c\left(A_{n}\right)\left(c_{1}\left(G_{n}^{(1)}\right) \omega-\operatorname{rk}\left(G_{n}^{(1)}\right) \beta \omega\right) .
\end{aligned}
$$

by (2.7). Since $c_{1}\left(A_{n}\right) \omega-\operatorname{rk}\left(A_{n}\right) \beta \omega>0$, we obtain

$$
\begin{aligned}
\frac{c\left(G_{n}^{(1)}\right)}{\operatorname{rk}\left(G_{n}^{(1)}\right)} \leq & \frac{c\left(A_{n}\right)}{\operatorname{rk}\left(A_{n}\right)} \cdot \frac{\mu_{\omega}\left(G_{n}^{(1)}\right)-\beta \omega}{\mu_{\omega}\left(A_{n}\right)-\beta \omega}+\frac{\omega^{2} m_{2, n}^{2}}{2} \cdot \frac{\mu_{\omega}\left(G_{n}^{(1)}\right)-\mu_{\omega}\left(A_{n}\right)}{\mu_{\omega}\left(A_{n}\right)-\beta \omega} \\
= & \frac{\omega^{2} m_{0, n}^{2}}{2} \cdot \frac{r \mu_{\omega}\left(A_{n}\right)-c_{1} \omega}{c_{1} \omega-r \beta \omega} \cdot \frac{\mu_{\omega}\left(G_{n}^{(1)}\right)-\beta \omega}{\mu_{\omega}\left(A_{n}\right)-\beta \omega} \\
& +c(\mathfrak{t}, \beta) \cdot \frac{\mu_{\omega}\left(G_{n}^{(1)}\right)-\beta \omega}{c_{1} \omega-r \beta \omega}+\frac{\omega^{2} m_{2, n}^{2}}{2} \cdot \frac{\mu_{\omega}\left(G_{n}^{(1)}\right)-\mu_{\omega}\left(A_{n}\right)}{\mu_{\omega}\left(A_{n}\right)-\beta \omega}
\end{aligned}
$$

where we have used (3.9) in the second step. Combining with (3.12), we get

$$
\begin{aligned}
-\frac{\epsilon_{n}^{2}}{2 \omega^{2}} \leq & \frac{\omega^{2} m_{0, n}^{2}}{2} \cdot \frac{r \mu_{\omega}\left(A_{n}\right)-c_{1} \omega}{c_{1} \omega-r \beta \omega} \cdot \frac{\mu_{\omega}\left(G_{n}^{(1)}\right)-\beta \omega}{\mu_{\omega}\left(A_{n}\right)-\beta \omega} \\
& +c(\mathfrak{t}, \beta) \cdot \frac{\mu_{\omega}\left(G_{n}^{(1)}\right)-\beta \omega}{c_{1} \omega-r \beta \omega}+\frac{\omega^{2} m_{2, n}^{2}}{2} \cdot \frac{\mu_{\omega}\left(G_{n}^{(1)}\right)-\mu_{\omega}\left(A_{n}\right)}{\mu_{\omega}\left(A_{n}\right)-\beta \omega} .
\end{aligned}
$$


If $m_{0, n}>m_{2, n}$ for all $n$, then since $\mu_{\omega}\left(G_{n}^{(1)}\right)-\mu_{\omega}\left(A_{n}\right) \geq 0$, we have

$$
\begin{aligned}
-\frac{\epsilon_{n}^{2}}{2 \omega^{2}} \leq & \frac{\omega^{2} m_{0, n}^{2}}{2} \cdot \frac{r \mu_{\omega}\left(A_{n}\right)-c_{1} \omega}{c_{1} \omega-r \beta \omega} \cdot \frac{\mu_{\omega}\left(G_{n}^{(1)}\right)-\beta \omega}{\mu_{\omega}\left(A_{n}\right)-\beta \omega} \\
& +\frac{\omega^{2} m_{0, n}^{2}}{2} \cdot \frac{\mu_{\omega}\left(G_{n}^{(1)}\right)-\mu_{\omega}\left(A_{n}\right)}{\mu_{\omega}\left(A_{n}\right)-\beta \omega}+c(\mathfrak{t}, \beta) \cdot \frac{\mu_{\omega}\left(G_{n}^{(1)}\right)-\beta \omega}{c_{1} \omega-r \beta \omega} \\
= & \frac{\omega^{2} m_{0, n}^{2}}{2} \cdot \frac{r \mu_{\omega}\left(G_{n}^{(1)}\right)-c_{1} \omega}{c_{1} \omega-r \beta \omega}+c(\mathfrak{t}, \beta) \cdot \frac{\mu_{\omega}\left(G_{n}^{(1)}\right)-\beta \omega}{c_{1} \omega-r \beta \omega} .
\end{aligned}
$$

Letting $n \rightarrow+\infty$, we obtain the contradiction $0 \leq-\omega^{2} m_{0}^{2} / 2$. Similarly, if $m_{0, n}<$ $m_{2, n}$ for all $n$, then $r \mu_{\omega}\left(A_{n}\right)-c_{1} \omega>0$ by (3.6) and (3.7). Therefore,

$$
\begin{aligned}
-\frac{\epsilon_{n}^{2}}{2 \omega^{2}} \leq & \frac{\omega^{2} m_{2, n}^{2}}{2} \cdot \frac{r \mu_{\omega}\left(A_{n}\right)-c_{1} \omega}{c_{1} \omega-r \beta \omega} \cdot \frac{\mu_{\omega}\left(G_{n}^{(1)}\right)-\beta \omega}{\mu_{\omega}\left(A_{n}\right)-\beta \omega} \\
& +\frac{\omega^{2} m_{2, n}^{2}}{2} \cdot \frac{\mu_{\omega}\left(G_{n}^{(1)}\right)-\mu_{\omega}\left(A_{n}\right)}{\mu_{\omega}\left(A_{n}\right)-\beta \omega}+c(\mathfrak{t}, \beta) \cdot \frac{\mu_{\omega}\left(G_{n}^{(1)}\right)-\beta \omega}{c_{1} \omega-r \beta \omega} \\
= & \frac{\omega^{2} m_{2, n}^{2}}{2} \cdot \frac{r \mu_{\omega}\left(G_{n}^{(1)}\right)-c_{1} \omega}{c_{1} \omega-r \beta \omega}+c(\mathfrak{t}, \beta) \cdot \frac{\mu_{\omega}\left(G_{n}^{(1)}\right)-\beta \omega}{c_{1} \omega-r \beta \omega} .
\end{aligned}
$$

Again, letting $n \rightarrow+\infty$, we obtain the contradiction $0 \leq-\omega^{2} m_{2}^{2} / 2$.

Proposition 3.10. The set of mini-walls is locally finite. More precisely, fix $\beta, \omega \in \operatorname{Num}(X)_{\mathbb{Q}}$ with $\omega$ being ample, $\mathfrak{t}=\left(r, c_{1}, c_{2}\right)$, and $I=[a, b]$ with $0<a<b$. Then there exist only finitely many mini-walls of type $(\mathfrak{t}, \beta, \omega)$ in $I$.

Proof. We may assume that $\beta, \omega \in \operatorname{Num}(X)$. Let $\mathbf{u}=e^{-(\beta+i \omega)}$, and let $m_{0}$ be a mini-wall of type $(\mathfrak{t}, \beta, \omega)$ in $I$. Then $\phi\left(Z_{m_{0}}(A)\right)=\phi\left(Z_{m_{0}}(E)\right)$ where $E \in \overline{\mathfrak{M}}_{\mathbf{u}_{m_{1}}}(\mathfrak{t})$ for some $m_{1} \in I, E \notin \overline{\mathfrak{M}}_{\mathbf{u}_{m_{2}}}(\mathfrak{t})$ for some $m_{2} \in I$, and $A$ is the leading HN-filtration component of $E$ with respect to $\left(Z_{m_{2}}, \mathcal{P}_{m_{2}}\right)$. So $A$ is $\left(Z_{m_{2}}, \mathcal{P}_{m_{2}}\right)$-semistable. By Lemma 3.9, $|\operatorname{rk}(A)| \leq N$ where $N$ depends only on $\mathfrak{t}, \beta, \omega$ and $I$.

Since $\phi\left(Z_{m_{0}}(A)\right)=\phi\left(Z_{m_{0}}(E)\right)$ and $A$ destablizes $E$ with respect to $\left(Z_{m_{2}}, \mathcal{P}_{m_{2}}\right)$,

$$
\begin{aligned}
& \frac{\omega^{2} m_{0}^{2}}{2}\left(r c_{1}(A) \omega-\operatorname{rk}(A) c_{1} \omega\right)=d(\mathfrak{t}, \beta, \omega, A) \\
& \frac{\omega^{2} m_{2}^{2}}{2}\left(r c_{1}(A) \omega-\operatorname{rk}(A) c_{1} \omega\right)>d(\mathfrak{t}, \beta, \omega, A) .
\end{aligned}
$$

So $\left(r c_{1}(A) \omega-\operatorname{rk}(A) c_{1} \omega\right) \neq 0$, and $m_{0}^{2}$ is equal to the rational number

$$
\frac{2 d(\mathfrak{t}, \beta, \omega, A)}{\omega^{2} \cdot\left(r c_{1}(A) \omega-\operatorname{rk}(A) c_{1} \omega\right)} \quad \in\left[a^{2}, b^{2}\right] .
$$

To prove that there are only finitely many choices for $m_{0}$, it suffices to show that the positive integer $\left|r c_{1}(A) \omega-\operatorname{rk}(A) c_{1} \omega\right|$ from the denominator is bounded from above by a number depending only on $\mathfrak{t}, \beta, \omega$ and $I$. Since $|\operatorname{rk}(A)| \leq N$, it remains to prove that there exist $N_{1}$ and $N_{2}$ depending only on $\mathfrak{t}, \beta, \omega$ and $I$ such that

$$
r N_{1} \leq r c_{1}(A) \omega \leq r N_{2} .
$$

Put $B=E / A$. Since $A, B \in \mathcal{A}_{(\omega, \beta \omega)}^{\sharp}$, we see from Lemma 3.6 (i) that

$$
c_{1}(A) \omega \geq \operatorname{rk}(A) \beta \omega, \quad c_{1}(B) \omega \geq \operatorname{rk}(B) \beta \omega .
$$


Note that (3.13) is trivially true if $r=0$. If $r<0$, then by (3.14),

$$
\begin{aligned}
r c_{1}(A) \omega & =r c_{1} \omega-r c_{1}(B) \omega \geq r c_{1} \omega-r \operatorname{rk}(B) \beta \omega \\
& =r c_{1} \omega-r(r-\operatorname{rk}(A)) \beta \omega \geq r c_{1} \omega-|r|(|r|+N)|\beta \omega| .
\end{aligned}
$$

In addition, we have $r c_{1}(A) \omega \leq r \operatorname{rk}(A) \beta \omega \leq|r| N|\beta \omega|$. Therefore, (3.13) holds for $r<0$. Similarly, we see that (3.13) holds for $r>0$ as well. $\square$

We remark that when $I=[a,+\infty)$ with $a>0$, the proof of Proposition 3.10 does not go through since it is unclear how to bound $|2 d(\mathfrak{t}, \beta, \omega, A)|$ from above.

4. Identify $\overline{\mathfrak{M}}_{\Omega}(\mathfrak{t})$ and $\overline{\mathfrak{M}}_{\mathbf{u}_{m}}(\mathfrak{t})$ for $m \gg 0$. In this section, we will strengthen Lemma 2.6. We show that there exists a constant $M$ depending only on $\mathfrak{t}(E), \omega$ and $\beta$ such that $E \in D^{b}(X)$ is $\left(Z_{\Omega}, \mathcal{P}_{\Omega}\right)$-semistable if and only if $E$ is $\left(Z_{m}, \mathcal{P}_{m}\right)$-semistable for some $m \geq M$.

Definition 4.1. If $E \in D^{b}(X)$ and $\beta, \omega \in \operatorname{Num}(X)_{\mathbb{R}}$ are fixed, then a constant is universal if it depends only on $\mathfrak{t}(E), \omega$ and $\beta$.

Lemma 4.2. Let notations be from Subsect. 2.2, and let $\omega \in \operatorname{Num}(X)_{\mathbb{Q}}$. If $E \in$ $D^{b}(X)$ is not $\left(Z_{\Omega}, \mathcal{P}_{\Omega}\right)$-semistable, then there exists a positive number $M$, depending only on $\mathfrak{t}(E), \omega$ and $\beta$, such that $E$ is not $\left(Z_{m}, \mathcal{P}_{m}\right)$-semistable for all $m \geq M$.

Proof. It suffices to prove the statement for $E \in \mathcal{P}_{\Omega}((0,1])=\mathcal{A}_{(\omega, \beta \omega)}^{\sharp}$. Let

$$
0 \rightarrow A \rightarrow E \rightarrow B \rightarrow 0
$$

be an exact sequence in $\mathcal{P}_{\Omega}((0,1])$ destablizing $E$ such that the object $B$ is $\left(Z_{\Omega}, \mathcal{P}_{\Omega}\right)$ semistable. Then $\phi\left(Z_{\Omega}(E)(m)\right)>\phi\left(Z_{\Omega}(B)(m)\right)$ for $m \gg 0$. So (2.7) holds for $m \gg 0$. By Lemma 3.6 (i), $c_{1}(E) \cdot \omega-\operatorname{rk}(E) \beta \omega \geq 0$ and $c_{1}(B) \cdot \omega-\operatorname{rk}(B) \beta \omega>0$. If $c_{1}(E) \cdot \omega-\operatorname{rk}(E) \beta \omega=0$, then $E$ is $\left(Z_{\Omega}, \mathcal{P}_{\Omega}\right)$-semistable which contradicts our assumption. So $c_{1}(E) \cdot \omega-\operatorname{rk}(E) \beta \omega>0$. Then, we have

$$
c_{1}(E) \cdot \omega-\operatorname{rk}(E) \beta \omega>0, \quad c_{1}(B) \cdot \omega-\operatorname{rk}(B) \beta \omega>0 .
$$

Now our proof is divided into three cases: $\operatorname{rk}(E)=0, \operatorname{rk}(E)>0$ and $\operatorname{rk}(E)<0$.

Case 1: $\operatorname{rk}(E)=0$. Then $c_{1}(E) \cdot \omega>0$ by (4.2). Since (2.7) holds for $m \gg 0$, we have $\operatorname{rk}(B) \geq 0$. If $\operatorname{rk}(B)=0$, then (2.7) holds for all $m>0$. So $B$ destablizes $E$ for all $m>0$, and we can take $M=1$. In the following, we assume that $\operatorname{rk}(B)>0$. By Lemma $2.4, B$ is a torsion free $\mu_{\omega}$-semistable sheaf with $\mu_{\omega}(B)>\beta \omega$. From (4.1), we obtain an exact sequence of sheaves $0 \rightarrow \mathcal{H}^{0}(A) \rightarrow \mathcal{H}^{0}(E) \rightarrow B \rightarrow 0$. So $\operatorname{rk}\left(\mathcal{H}^{0}(E)\right)>$ 0 . Going backwards, let $\widetilde{B}$ to be the HN-filtration quotient of $\mathcal{H}^{0}(E)$ with smallest $\mu_{\omega}$-slope. Then, $\mu_{\omega}\left(\mathcal{H}^{0}(E) / \operatorname{Tor}\left(\mathcal{H}^{0}(E)\right)\right) \geq \mu_{\omega}(\widetilde{B})$ and $\widetilde{B}$ is $\mu_{\omega}$-semistable. Since $\mathcal{H}^{0}(E) \in \mathcal{T}_{(\omega, \beta \omega)}$, we also have $\mu_{\omega}(\widetilde{B})>\beta \omega$. Therefore,

$$
\mu_{\omega}\left(\mathcal{H}^{0}(E) / \operatorname{Tor}\left(\mathcal{H}^{0}(E)\right)\right) \geq \mu_{\omega}(\widetilde{B})>\beta \omega .
$$

By Lemma 3.5 (i), we have an exact sequence $0 \rightarrow \widetilde{A} \rightarrow E \rightarrow \widetilde{B} \rightarrow 0$ in $\mathcal{A}_{(\omega, \beta \omega)}^{\sharp}$ which destablizes $E$ in view of (2.7) (replace $B$ there by $\widetilde{B}$ ). Hence, replacing $B$ in (4.1) by $\widetilde{B}$, we may assume in (4.1) that $B=B[0]$ satisfies:

$$
\mu_{\omega}\left(\mathcal{H}^{0}(E) / \operatorname{Tor}\left(\mathcal{H}^{0}(E)\right)\right) \geq \mu_{\omega}(B)>\beta \omega .
$$


Note that $\operatorname{rk}\left(\mathcal{H}^{-1}(E)\right)=\operatorname{rk}\left(\mathcal{H}^{0}(E)\right)>0$. Since $\mathcal{H}^{-1}(E) \in \mathcal{F}_{(\omega, \beta \omega)}, \mu_{\omega}\left(\mathcal{H}^{-1}(E)\right) \leq$ $\beta \omega$. Since $c_{1}\left(\mathcal{H}^{0}(E)\right)=c_{1}(E)+c_{1}\left(\mathcal{H}^{-1}(E)\right)$ and $c_{1}(E) \cdot \omega>0$, we have

$$
\begin{aligned}
\mu_{\omega}\left(\mathcal{H}^{0}(E) / \operatorname{Tor}\left(\mathcal{H}^{0}(E)\right)\right) & \leq \mu_{\omega}\left(\mathcal{H}^{0}(E)\right)=\frac{c_{1}\left(\mathcal{H}^{0}(E)\right) \cdot \omega}{\operatorname{rk}\left(\mathcal{H}^{0}(E)\right)} \\
& =\frac{\left(c_{1}(E)+c_{1}\left(\mathcal{H}^{-1}(E)\right)\right) \cdot \omega}{\operatorname{rk}\left(\mathcal{H}^{-1}(E)\right)} \leq c_{1}(E) \cdot \omega+\beta \omega .
\end{aligned}
$$

Combining with (4.4), $\left(c_{1}(E) \cdot \omega+\beta \omega\right) \geq \mu_{\omega}(B)>\beta \omega$. By Lemma 3.7 (ii), $c(B) / \operatorname{rk}(B)$ is bounded from below by a universal constant. By (2.7), there exists a constant $M$, depending only on $\mathfrak{t}(E), \omega$ and $\beta$, such that whenever $m \geq M, \phi\left(Z_{\Omega}(E)(m)\right)>$ $\phi\left(Z_{\Omega}(B)(m)\right)$ and so $E$ is not $\left(Z_{m}, \mathcal{P}_{m}\right)$-semistable.

Case 2: $\operatorname{rk}(E)>0$. Then $\mu_{\omega}(E)>\beta \omega$ by $(4.2)$, and $\operatorname{rk}\left(\mathcal{H}^{0}(E)\right)>0$. Let $\mathcal{E}=\mathcal{H}^{-1}(E)$. Assume that $\mathcal{E} \neq 0$. Then $\mu_{\omega}(\mathcal{E}) \leq \beta \omega$ since $\mathcal{E} \in \mathcal{F}_{(\omega, \beta \omega)}$. As in Case 1 , we can choose the object $B$ in (4.1) to be the HN-filtration quotient of $\mathcal{H}^{0}(E)$ with smallest $\mu_{\omega}$-slope. Then $B$ is semistable and satisfies (4.4). By (4.4),

$$
\begin{aligned}
\mu_{\omega}(B)-\mu_{\omega}(E) & \leq \mu_{\omega}\left(\mathcal{H}^{0}(E)\right)-\mu_{\omega}(E)=\frac{\left(c_{1}(E)+c_{1}(\mathcal{E})\right) \omega}{\operatorname{rk}(E)+\operatorname{rk}(\mathcal{E})}-\mu_{\omega}(E) \\
& =\frac{\mu_{\omega}(\mathcal{E})-\mu_{\omega}(E)}{1+\operatorname{rk}(E) / \operatorname{rk}(\mathcal{E})} \leq \frac{\beta \omega-\mu_{\omega}(E)}{1+\operatorname{rk}(E) / \operatorname{rk}(\mathcal{E})} \\
& \leq \frac{\beta \omega-\mu_{\omega}(E)}{1+\operatorname{rk}(E)}<0
\end{aligned}
$$

So $\mu_{\omega}(E)>\mu_{\omega}(B)>\beta \omega$. By Lemma 3.7 (ii), $c(B) / \operatorname{rk}(B)$ is bounded from below by a universal constant. Now (2.7) is equivalent to

$$
\frac{\omega^{2} m^{2}}{2}\left(\mu_{\omega}(B)-\mu_{\omega}(E)\right)<\frac{c(B)}{\operatorname{rk}(B)}\left(\mu_{\omega}(E)-\beta \omega\right)-\frac{c(E)}{\operatorname{rk}(E)}\left(\mu_{\omega}(B)-\beta \omega\right) .
$$

In view of the negative upper bound $\left(\beta \omega-\mu_{\omega}(E)\right) /(1+\operatorname{rk}(E))$ for $\left(\mu_{\omega}(B)-\mu_{\omega}(E)\right)$ from (4.5), there exists a constant $M$, depending only on $\mathfrak{t}(E), \omega$ and $\beta$, such that $\phi\left(Z_{\Omega}(E)(m)\right)>\phi\left(Z_{\Omega}(B)(m)\right)$ whenever $m \geq M$. Hence our lemma holds.

Let $\mathcal{E}=0$. Then $E=\mathcal{H}^{0}(E)$ has positive rank. If Tor $(E)$ contains a 0-dimensional subsheaf $Q$, then $Q \in \mathcal{A}_{(\omega, \beta \omega)}^{\sharp}$ is a proper sub-object of $E$ destablizing $E$ with respect to $\left(Z_{m}, \mathcal{P}_{m}\right)$ for all $m>0$ and we are done. If $\operatorname{Tor}(E)$ is a 1-dimensional torsion, then we can choose $B$ in (4.1) to be the HN-filtration quotient of $E$ with smallest $\mu_{\omega}$-slope. Now $B$ is $\mu_{\omega}$-semistable and satisfies

$$
\beta \omega<\mu_{\omega}(B) \leq \mu_{\omega}(E / \operatorname{Tor}(E)) \leq \mu_{\omega}(E)-1 / \operatorname{rk}(E) .
$$

So $\mu_{\omega}(B)-\mu_{\omega}(E) \leq-1 / \operatorname{rk}(E)$. Again $c(B) / \operatorname{rk}(B)$ is bounded from below by a universal constant. In view of (4.6), our lemma holds. In the following, assume that $\operatorname{Tor}(E)=0$. Let $\widetilde{B}$ be the HN-filtration quotient of $E$ with smallest $\mu_{\omega}$-slope. Then $\widetilde{B}$ is $\mu_{\omega}$-semistable and satisfies the inequalities $\mu_{\omega}(E) \geq \mu_{\omega}(\widetilde{B})>\beta \omega$. If $\mu_{\omega}(E)>\mu_{\omega}(\widetilde{B})$, then we can choose the object $B$ in (4.1) such that $B=\widetilde{B}$. Since $\operatorname{rk}(B)<\operatorname{rk}(E)$, the rational number $\mu_{\omega}(B)-\mu_{\omega}(E)$ is bounded from above by a negative universal constant. Hence in view of (4.6), our lemma holds. We are left with the case when $\mu_{\omega}(E)=\mu_{\omega}(\widetilde{B})$, i.e., $E=\widetilde{B}$ is $\mu_{\omega}$-semistable with $\mu_{\omega}(E)>\beta \omega$. 
We claim that this is impossible. Indeed, we see from (4.1) that $A \neq 0$ is a torsion free sheaf and sits in the exact sequence

$$
0 \rightarrow \mathcal{H}^{-1}(B) \rightarrow A \rightarrow E \rightarrow \mathcal{H}^{0}(B) \rightarrow 0 .
$$

Since $\phi\left(Z_{\Omega}(E)(m)\right)<\phi\left(Z_{\Omega}(A)(m)\right)$ for $m \gg 0$, we see from Remark 2.8 that

$$
\frac{\omega^{2} m^{2}}{2}\left(\mu_{\omega}(A)-\mu_{\omega}(E)\right)>\frac{c(A)}{\operatorname{rk}(A)}\left(\mu_{\omega}(E)-\beta \omega\right)-\frac{c(E)}{\operatorname{rk}(E)}\left(\mu_{\omega}(A)-\beta \omega\right)
$$

for $m \gg 0$. So $\mu_{\omega}(A) \geq \mu_{\omega}(E)$. If $\mu_{\omega}(A)=\mu_{\omega}(E)$, then (4.8) holds for all $m>0$ and our lemma holds by taking $M=1$. In the following, assume that $\mu_{\omega}(A)>\mu_{\omega}(E)$. Since $E$ is $\mu_{\omega}$-semistable, $\mathcal{B}:=\mathcal{H}^{-1}(B) \neq 0$ by (4.7). By Lemma $2.4, \mathcal{B}$ is $\mu_{\omega^{-}}$ semistable with $\mu_{\omega}(\mathcal{B}) \leq \beta \omega$, and $\mathcal{H}^{0}(B)$ is a 0 -dimensional torsion sheaf. Let $\mathcal{G}$ be the image of the map $A \rightarrow E$ from (4.7). Then we have two exact sequences of sheaves:

$$
\begin{gathered}
0 \rightarrow \mathcal{B} \rightarrow A \rightarrow \mathcal{G} \rightarrow 0, \\
0 \rightarrow \mathcal{G} \rightarrow E \rightarrow \mathcal{H}^{0}(B) \rightarrow 0 .
\end{gathered}
$$

By $(4.10), \mu_{\omega}(\mathcal{G})=\mu_{\omega}(E)<\mu_{\omega}(A)$. So $\mu_{\omega}(\mathcal{B})>\mu_{\omega}(A)$ by $(4.9)$. However, this contradicts $\mu_{\omega}(\mathcal{B}) \leq \beta \omega<\mu_{\omega}(E)<\mu_{\omega}(A)$.

Case 3: $\operatorname{rk}(E)<0$. Let $\mathcal{E}=\mathcal{H}^{-1}(E)$. Then $\mu_{\omega}(E)<\beta \omega$ by $(4.2)$, and $\mathcal{E} \neq 0$ is torsion free. Assume that $\operatorname{rk}\left(\mathcal{H}^{0}(E)\right)>0$. As in Case 1, we can choose the object $B$ in (4.1) to be the HN-filtration quotient of $\mathcal{H}^{0}(E)$ with smallest $\mu_{\omega}$-slope. Then $B$ is $\mu_{\omega}$-semistable and satisfies (4.4), and $\mu_{\omega}(\mathcal{E}) \leq \beta \omega$ since $\mathcal{E} \in \mathcal{F}_{(\omega, \beta \omega)}$. By (4.4),

$$
\begin{aligned}
\mu_{\omega}(B)-\mu_{\omega}(E) & \leq \mu_{\omega}\left(\mathcal{H}^{0}(E)\right)-\mu_{\omega}(E) \\
& =\frac{\mu_{\omega}(\mathcal{E})-\mu_{\omega}(E)}{\operatorname{rk}\left(\mathcal{H}^{0}(E)\right) / \operatorname{rk}(\mathcal{E})} \\
& \leq\left(\beta \omega-\mu_{\omega}(E)\right) \cdot \frac{\operatorname{rk}(\mathcal{E})}{\operatorname{rk}\left(\mathcal{H}^{0}(E)\right)} \\
& =\left(\beta \omega-\mu_{\omega}(E)\right) \cdot\left(1-\frac{\operatorname{rk}(E)}{\operatorname{rk}\left(\mathcal{H}^{0}(E)\right)}\right) \\
& \leq\left(\beta \omega-\mu_{\omega}(E)\right) \cdot(1-\operatorname{rk}(E)) .
\end{aligned}
$$

Combining with (4.4), we conclude that

$$
\mu_{\omega}(E)+\left(\beta \omega-\mu_{\omega}(E)\right) \cdot(1-\operatorname{rk}(E)) \geq \mu_{\omega}(B)>\beta \omega .
$$

So $c(B) / \operatorname{rk}(B)$ is bounded from below by a constant depending only on $\mathfrak{t}(E), \omega$ and $\beta$. Also, $\mu_{\omega}(B)-\mu_{\omega}(E)>\beta \omega-\mu_{\omega}(E)>0$. Now (2.7) is equivalent to

(4.13) $\frac{\omega^{2} m^{2}}{2}\left(\mu_{\omega}(B)-\mu_{\omega}(E)\right)>\frac{c(B)}{\operatorname{rk}(B)}\left(\mu_{\omega}(E)-\beta \omega\right)-\frac{c(E)}{\operatorname{rk}(E)}\left(\mu_{\omega}(B)-\beta \omega\right)$.

It follows that there exists a constant $M$, depending only on $\mathfrak{t}(E), \omega$ and $\beta$, such that $E$ is not $\left(Z_{m}, \mathcal{P}_{m}\right)$-semistable whenever $m \geq M$.

We are left with the case $\operatorname{rk}\left(\mathcal{H}^{0}(E)\right)=0$. Assume that either $\mathcal{H}^{-1}(E)$ is $\mu_{\omega^{-}}$ unstable, or the support of $\mathcal{H}^{0}(E)$ has dimension 1 . Let $A$ be the HN-filtration 
subsheaf of $\mathcal{H}^{-1}(E)$ with largest $\mu_{\omega}$-slope. Then $A \in \mathcal{F}_{(\omega, \beta \omega)}$ is $\mu_{\omega}$-semistable with $\mu_{\omega}(A) \leq \beta \omega$. When $\mathcal{H}^{-1}(E)$ is $\mu_{\omega}$-unstable, $\mu_{\omega}(A)>\mu_{\omega}\left(\mathcal{H}^{-1}(E)\right)$; so $\mu_{\omega}(A) \geq$ $\mu_{\omega}\left(\mathcal{H}^{-1}(E)\right)+d_{1}$ for some positive number $d_{1}$ depending only on $\operatorname{rk}(E)$ and $\omega$. When the support of $\mathcal{H}^{0}(E)$ has dimension 1, we have

$$
\begin{aligned}
\mu_{\omega}(A) & \geq \mu_{\omega}\left(\mathcal{H}^{-1}(E)\right)=\frac{\left(c_{1}\left(\mathcal{H}^{0}(E)\right)-c_{1}(E)\right) \cdot \omega}{\operatorname{rk} \mathcal{H}^{-1}(E)} \\
& \geq \frac{1-c_{1}(E) \cdot \omega}{-\operatorname{rk}(E)}=\mu_{\omega}(E)-\frac{1}{\operatorname{rk}(E)}
\end{aligned}
$$

In either case, $\beta \omega \geq \mu_{\omega}(A) \geq \mu_{\omega}(E)+d_{2}$ where $d_{2}$ is a positive number depending only on $\operatorname{rk}(E)$ and $\omega$. In particular, $\mu_{\omega}(A)-\mu_{\omega}(E) \geq d_{2}$. Since $\mu_{\omega}(E)<\beta \omega$, we see from Lemma 3.7 (ii) that $c(A) / \operatorname{rk}(A) \cdot\left(\mu_{\omega}(E)-\beta \omega\right)$ is bounded from above by a constant depending only on $\mathfrak{t}(E), \omega$ and $\beta$. Hence there exists $M$ depending only on $\mathfrak{t}(E), \omega$ and $\beta$ such that (4.8) holds whenever $m \geq M$. By Remark 2.8, $\phi\left(Z_{\Omega}(E)(m)\right)<\phi\left(Z_{\Omega}(A[1])(m)\right)$ whenever $m \geq M$. By Lemma 3.5 (ii), $A[1]$ is a proper sub-object of $E$ in $\mathcal{A}_{(\omega, \beta \omega)}^{\sharp}$. So $A[1]$ destablizes $E$ whenever $m \geq M$.

Finally, assume that $\mathcal{H}^{-1}(E)$ is $\mu_{\omega}$-semistable and $\mathcal{H}^{0}(E)$ is a 0 -dimensional torsion sheaf. By the exact sequence of sheaves

$$
0 \rightarrow \mathcal{H}^{-1}(A) \rightarrow \mathcal{E} \rightarrow \mathcal{B} \rightarrow \mathcal{H}^{0}(A) \rightarrow \mathcal{H}^{0}(E) \rightarrow \mathcal{H}^{0}(B) \rightarrow 0,
$$

$\mathcal{H}^{0}(B)$ is a 0 -dimensional torsion sheaf. Since $B$ destablizes $E$ with respect to $\left(Z_{\Omega}, \mathcal{P}_{\Omega}\right), B$ can not be a 0 -dimensional torsion sheaf. By Lemma $2.4, \mathcal{B}:=\mathcal{H}^{-1}(B)$ is a torsion free $\mu_{\omega}$-semistable sheaf with $\mu_{\omega}(\mathcal{B}) \leq \beta \omega$. Since $\mu_{\omega}(E)=\mu_{\omega}(\mathcal{E})$ and $\mu_{\omega}(B)=\mu_{\omega}(\mathcal{B}),(2.7)$ is equivalent to

$$
\frac{\omega^{2} m^{2}}{2}\left(\mu_{\omega}(\mathcal{B})-\mu_{\omega}(\mathcal{E})\right)<\frac{c(B)}{\operatorname{rk}(B)}\left(\mu_{\omega}(\mathcal{E})-\beta \omega\right)-\frac{c(E)}{\operatorname{rk}(E)}\left(\mu_{\omega}(\mathcal{B})-\beta \omega\right) .
$$

Since it holds for $m \gg 0, \mu_{\omega}(\mathcal{B}) \leq \mu_{\omega}(\mathcal{E})$. If $\mu_{\omega}(\mathcal{B})=\mu_{\omega}(\mathcal{E})$, then $(4.15)$ holds for all $m \geq 1$; so our lemma is true with $M=1$. Let $\mu_{\omega}(\mathcal{B})<\mu_{\omega}(\mathcal{E})$. Then $\mu_{\omega}(\mathcal{B})<\mu_{\omega}(\mathcal{E})<\beta \omega$. Since $\mathcal{E}$ and $\mathcal{B}$ are $\mu_{\omega}$-semistable, the map $\mathcal{E} \rightarrow \mathcal{B}$ in $(4.14)$ is zero. So we obtain the exact sequence $0 \rightarrow \mathcal{B} \rightarrow \mathcal{H}^{0}(A) \rightarrow \mathcal{H}^{0}(E) \rightarrow \mathcal{H}^{0}(B) \rightarrow 0$. Since $\mathcal{H}^{0}(A) \in \mathcal{T}_{(\omega, \beta \omega)}$, we get the contradiction

$$
\beta \omega<\mu_{\omega}\left(\mathcal{H}^{0}(A)\right)=\mu_{\omega}(\mathcal{B})<\beta \omega .
$$

Lemma 4.3. Let notations be from Subsect. 2.2, and let $\omega \in \operatorname{Num}(X)_{\mathbb{Q}}$. If an object $E \in D^{b}(X)$ is $\left(Z_{\Omega}, \mathcal{P}_{\Omega}\right)$-semistable, then there exists a positive $M$, depending only on $\mathfrak{t}(E), \omega$ and $\beta$, such that $E$ is $\left(Z_{m}, \mathcal{P}_{m}\right)$-semistable for all $m \geq M$.

Proof. It suffices to prove the statement for $E \in \mathcal{P}_{\Omega}((0,1])=\mathcal{A}_{(\omega, \beta \omega)}^{\sharp}$. We begin with an observation. Consider the set

$$
W=\left\{w \in[1,+\infty) \mid E \text { is }\left(Z_{w}, \mathcal{P}_{w}\right) \text {-unstable }\right\} .
$$

If $W$ is empty, then we are done by taking $M=1$. Assume that $W$ is nonempty. By Lemma 2.6, $E$ is $\left(Z_{m}, \mathcal{P}_{m}\right)$-semistable for $m \gg 0$. So for every $w \in W$, we can find a maximal destablizing sub-object $A_{w} \in \mathcal{A}_{(\omega, \beta \omega)}^{\sharp}$ of $E$ with respect to $\left(Z_{w}, \mathcal{P}_{w}\right)$, 
satisfying the properties listed in Lemma 3.9. By Lemma 3.9, there exists a universal constant $N$ (depending only on $\mathfrak{t}(E), \beta$ and $\omega$ ) such that

$$
\left|\operatorname{rk}\left(A_{w}\right)\right| \leq N
$$

We need to show that $W$ has a universal upper bound. To show this, it suffices to prove that, given any exact sequence in $\mathcal{A}_{(\omega, \beta \omega)}^{\sharp}$ :

$$
0 \rightarrow A_{w} \rightarrow E \rightarrow B_{w} \rightarrow 0
$$

where $E$ is $\left(Z_{w}, \mathcal{P}_{w}\right)$-unstable for some $w \in W$ and $A_{w}$ is the maximal destablizing sub-object with respect to $\left(Z_{w}, \mathcal{P}_{w}\right)$, we can find a constant $M>0$ depending only on $\mathfrak{t}(E), \omega$ and $\beta$ such that $\phi\left(Z_{\Omega}\left(A_{w}\right)(m)\right) \leq \phi\left(Z_{\Omega}(E)(m)\right)$ whenever $m>M$, i.e.,

$$
\begin{aligned}
& \frac{\omega^{2} m^{2}}{2}\left(\operatorname{rk}(E) c_{1}\left(A_{w}\right) \omega-\operatorname{rk}\left(A_{w}\right) c_{1}(E) \omega\right) \\
\leq & c\left(A_{w}\right)\left(c_{1}(E) \omega-\operatorname{rk}(E) \beta \omega\right)-c(E)\left(c_{1}\left(A_{w}\right) \omega-\operatorname{rk}\left(A_{w}\right) \beta \omega\right) .
\end{aligned}
$$

whenever $m \geq M$, in view of the discussions in Remark 2.8. So fix such an exact sequence (4.19). Note that $E$ satisfies Lemma 2.4 (i), (ii) or (iii). In the following, our proof is divided into three cases accordingly.

Case 1: $E$ satisfies Lemma 2.4 (i). If $E$ is a 0 -dimensional torsion sheaf, then it is $\left(Z_{m}, \mathcal{P}_{m}\right)$-semistable for all $m>0$, contradicting the nonemptiness of $W$. So $E$ must be a 1-dimensional torsion sheaf, and (4.20) is simplified to

$$
\frac{\omega^{2} m^{2}}{2}\left(-\operatorname{rk}\left(A_{w}\right) c_{1}(E) \omega\right) \leq c\left(A_{w}\right) c_{1}(E) \omega-c(E)\left(c_{1}\left(A_{w}\right) \omega-\operatorname{rk}\left(A_{w}\right) \beta \omega\right)
$$

Note from the long exact sequence of cohomology of (4.19) that $A_{w}$ is a sheaf and

$$
0 \rightarrow \mathcal{H}^{-1}\left(B_{w}\right) \rightarrow A_{w} \rightarrow E \rightarrow \mathcal{H}^{0}\left(B_{w}\right) \rightarrow 0
$$

is an exact sequence of sheaves. Since (4.21) holds for $m \gg 0$ but does not hold for $m=w, \operatorname{rk}\left(A_{w}\right)>0$. By (4.18), $0<\operatorname{rk}\left(A_{w}\right) \leq N$. Since $\operatorname{rk}\left(\mathcal{H}^{-1}\left(B_{w}\right)\right)=\operatorname{rk}\left(A_{w}\right)$, $0<\operatorname{rk}\left(\mathcal{H}^{-1}\left(B_{w}\right)\right) \leq N$. By the definition of $\mathcal{A}_{(\omega, \beta \omega)}^{\sharp}$, we have $\mu_{\omega}\left(A_{w}\right)>\beta \omega$. So

$$
c_{1}\left(A_{w}\right) \omega>\operatorname{rk}\left(A_{w}\right) \cdot \beta \omega \geq-N \cdot|\beta \omega| .
$$

Similarly, we have $\mu_{\omega}\left(\mathcal{H}^{-1}\left(B_{w}\right)\right) \leq \beta \omega$. It follows that

$$
c_{1}\left(\mathcal{H}^{-1}\left(B_{w}\right)\right) \omega \leq \operatorname{rk}\left(\mathcal{H}^{-1}\left(B_{w}\right)\right) \cdot \beta \omega \leq N \cdot|\beta \omega| .
$$

Note that $\mathcal{H}^{0}\left(B_{w}\right)$ is a torsion sheaf. Thus $c_{1}\left(\mathcal{H}^{0}\left(B_{w}\right)\right) \geq 0$ and

$$
c_{1}\left(B_{w}\right) \omega=c_{1}\left(\mathcal{H}^{0}\left(B_{w}\right)\right) \omega-c_{1}\left(\mathcal{H}^{-1}\left(B_{w}\right)\right) \omega \geq-c_{1}\left(\mathcal{H}^{-1}\left(B_{w}\right)\right) \omega \geq-N \cdot|\beta \omega| \text {. }
$$

Since $c_{1}\left(A_{w}\right)=c_{1}(E)-c_{1}\left(B_{w}\right)$, we see from (4.23) and (4.24) that

$$
-N \cdot|\beta \omega| \leq c_{1}\left(A_{w}\right) \omega \leq c_{1}(E) \omega+N \cdot|\beta \omega| .
$$

So $\operatorname{rk}\left(A_{w}\right),\left|c_{1}\left(A_{w}\right) \omega\right|$ and $\left|\mu_{\omega}\left(A_{w}\right)\right|$ are bounded from above by universal constants. 
Consider the usual HN-filtration of the sheaf $A_{w}$ with respect to $\mu_{\omega}$ :

$$
\operatorname{Tor}\left(A_{w}\right)=A_{0} \subset A_{1} \subset \cdots \subset A_{n}=A_{w}
$$

where $n \leq \operatorname{rk}\left(A_{w}\right) \leq N$. Then $\mu_{\omega}\left(A_{w}\right) \geq \mu_{\omega}\left(A_{n} / A_{n-1}\right)$. By the definition of $\mathcal{A}_{(\omega, \beta \omega)}^{\sharp}$, we have $\mu_{\omega}\left(A_{n} / A_{n-1}\right)>\beta \omega$. Hence $\operatorname{rk}\left(A_{n-1}\right),\left|c_{1}\left(A_{n-1}\right) \omega\right|,\left|\mu_{\omega}\left(A_{n-1}\right)\right|$ and $\left|\mu_{\omega}\left(A_{n} / A_{n-1}\right)\right|$ are bounded from above by universal constants. Similarly, using $A_{n-1}$ instead of $A_{n}=A_{w}$, we see that $\operatorname{rk}\left(A_{n-2}\right),\left|c_{1}\left(A_{n-2}\right) \omega\right|,\left|\mu_{\omega}\left(A_{n-2}\right)\right|$ and $\left|\mu_{\omega}\left(A_{n-1} / A_{n-2}\right)\right|$ are bounded from above by universal constants. Repeating this process, we conclude that $\operatorname{rk}\left(A_{i}\right),\left|c_{1}\left(A_{i}\right) \omega\right|,\left|\mu_{\omega}\left(A_{i}\right)\right|$ and $\left|\mu_{\omega}\left(A_{i} / A_{i-1}\right)\right|$, with $1 \leq i \leq n$, are all bounded from above by a universal constant. Applying Lemma 3.7 (ii) to the torsion free $\mu_{\omega}$-semistable sheaves $A_{i} / A_{i-1}$, we see that all the numbers $c\left(A_{i} / A_{i-1}\right)$, $1 \leq i \leq n$, are bounded from below by a universal constant. Suppose $\operatorname{Tor}\left(A_{w}\right) \neq 0$. To understand $c\left(A_{0}\right)=c\left(\operatorname{Tor}\left(A_{w}\right)\right)$, note from (4.22) that $\operatorname{Tor}\left(A_{w}\right)$ does not contain any 0-dimensional subsheaf because $E$ is $\left(Z_{\Omega}, \mathcal{P}_{\Omega}\right)$-semistable, $\operatorname{rk}(E)=0$ and $c_{1}(E)>0$. So $\operatorname{Tor}\left(A_{w}\right)$ is a 1-dimensional torsion sheaf. Since $H^{-1}\left(B_{w}\right)$ is torsion free, the subsheaf $\operatorname{Tor}\left(A_{w}\right)$ of $A_{w}$ is mapped injectively into $E$. Therefore, $0<c_{1}\left(\operatorname{Tor}\left(A_{w}\right)\right) \omega \leq c_{1}(E) \omega$. Note that the sheaf injection $\operatorname{Tor}\left(A_{w}\right) \hookrightarrow A_{w}$ is also an injection in $\mathcal{A}_{(\omega, \beta \omega)}^{\sharp}$. So $\operatorname{Tor}\left(A_{w}\right)$ is a sub-object of $E$ in $\mathcal{A}_{(\omega, \beta \omega)}^{\sharp}$. By the $\left(Z_{\Omega}, \mathcal{P}_{\Omega}\right)$ semistability of $E$ and using (2.7), we see that $c\left(\operatorname{Tor}\left(A_{w}\right)\right)$ is bounded from below by a universal constant. Overall, we have proved that

$$
c\left(A_{0}\right), \quad c\left(A_{i} / A_{i-1}\right),
$$

with $1 \leq i \leq n$, are bounded from below by a universal constant. Note from (2.6) that $c\left(A_{w}\right)=c\left(A_{0}\right)+\sum_{i=1}^{n} c\left(A_{i} / A_{i-1}\right)$. Since $n \leq N, c\left(A_{w}\right)$ is bounded from below by a universal constant. Hence $c\left(A_{w}\right) / \operatorname{rk}\left(A_{w}\right)$ is bounded from below by a universal constant. By (4.21), there exists a universal constant $M$ such that $\phi\left(Z_{\Omega}\left(A_{w}\right)(m)\right) \leq$ $\phi\left(Z_{\Omega}(E)(m)\right)$ whenever $m \geq M$.

Case 2: $E$ satisfies Lemma 2.4 (ii). We see from the exact sequence (4.19) that $A_{w}$ is a torsion free sheaf. So (4.20) is equivalent to

$$
\frac{\omega^{2} m^{2}}{2}\left(\mu_{\omega}\left(A_{w}\right)-\mu_{\omega}(E)\right) \leq \frac{c\left(A_{w}\right)}{\operatorname{rk}\left(A_{w}\right)}\left(\mu_{\omega}(E)-\beta \omega\right)-\frac{c(E)}{\operatorname{rk}(E)}\left(\mu_{\omega}\left(A_{w}\right)-\beta \omega\right) .
$$

Since (4.25) holds for $m \gg 0$ but does not hold for $m=w$, we must have $\mu_{\omega}\left(A_{w}\right)<$ $\mu_{\omega}(E)$. Since $A_{w} \in \mathcal{A}_{(\omega, \beta \omega)}^{\sharp}$, we get $\mu_{\omega}\left(A_{w}\right)>\beta \omega$. Thus, $\beta \omega<\mu_{\omega}\left(A_{w}\right)<\mu_{\omega}(E)$. By (4.18), $\operatorname{rk}\left(A_{w}\right)$ is bounded from above by a universal number. Hence the negative rational number $\left(\mu_{\omega}\left(A_{w}\right)-\mu_{\omega}(E)\right)$ has a universal negative upper bound. Next, consider the HN-filtration of the torsion free sheaf $A_{w}$ with respect to $\mu_{\omega}$ :

$$
0=A_{0} \subset A_{1} \subset \cdots \subset A_{n}=A_{w} .
$$

Since $\operatorname{rk}\left(A_{w}\right),\left|c_{1}\left(A_{w}\right) \omega\right|$ and $\left|\mu_{\omega}\left(A_{w}\right)\right|$ are bounded from above by universal constants, the same argument as in the previous paragraph proves that $c\left(A_{w}\right) / \operatorname{rk}\left(A_{w}\right)$ is bounded from below by a universal constant. By (4.25), there exists a universal constant $M$ such that $\phi\left(Z_{\Omega}\left(A_{w}\right)(m)\right) \leq \phi\left(Z_{\Omega}(E)(m)\right)$ whenever $m \geq M$.

Case 3: $E$ satisfies Lemma 2.4 (iii). Note that (4.20) is equivalent to

$$
\begin{aligned}
& \frac{\omega^{2} m^{2}}{2}\left(\operatorname{rk}(E) c_{1}\left(B_{w}\right) \omega-\operatorname{rk}\left(B_{w}\right) c_{1}(E) \omega\right) \\
\geq & c\left(B_{w}\right)\left(c_{1}(E) \omega-\operatorname{rk}(E) \beta \omega\right)-c(E)\left(c_{1}\left(B_{w}\right) \omega-\operatorname{rk}\left(B_{w}\right) \beta \omega\right) .
\end{aligned}
$$


Since $\mathcal{H}^{0}(E)$ is a 0 -dimensional torsion sheaf, so is $\mathcal{H}^{0}\left(B_{w}\right)$. Put $\mathcal{B}=\mathcal{H}^{-1}\left(B_{w}\right)$. Since (4.26) holds for $m \gg 0$ but does not hold for $m=w, B_{w}$ can not be a 0-dimensional torsion sheaf. In particular, $B_{w} \neq \mathcal{H}^{0}\left(B_{w}\right)$. So $\mathcal{B} \neq 0$. Note that $\mathcal{B}$ is torsion free. Now the inequality (4.26) is equivalent to

$$
\frac{\omega^{2} m^{2}}{2}\left(\mu_{\omega}(\mathcal{B})-\mu_{\omega}(E)\right) \geq \frac{c\left(B_{w}\right)}{\operatorname{rk}(\mathcal{B})}\left(\beta \omega-\mu_{\omega}(E)\right)-\frac{c(E)}{\operatorname{rk}(E)}\left(\mu_{\omega}(\mathcal{B})-\beta \omega\right) .
$$

Since (4.27) holds for $m \gg 0$ but does not hold for $m=w, \mu_{\omega}(\mathcal{B})>\mu_{\omega}(E)$. Since $E, B_{w} \in \mathcal{A}_{(\omega, \beta \omega)}^{\sharp}$, we have $\mu_{\omega}(E), \mu_{\omega}\left(B_{w}\right) \leq \beta \omega$ by Lemma 3.6 (i). Thus,

$$
\beta \omega \geq \mu_{\omega}(\mathcal{B})>\mu_{\omega}(E)
$$

By (4.18), $\left|\operatorname{rk}\left(A_{w}\right)\right|$ is bounded from above by a universal constant. So

$$
\operatorname{rk}(\mathcal{B})=\left|\operatorname{rk}\left(B_{w}\right)\right| \leq|\operatorname{rk}(E)|+\left|\operatorname{rk}\left(A_{w}\right)\right|
$$

is bounded from above by a universal constant. Thus the positive rational number $\left(\mu_{\omega}(\mathcal{B})-\mu_{\omega}(E)\right)$ has a universal positive lower bound. In view of $(4.27)$, to prove our lemma, it remains to show that there exists a universal constant $\widetilde{N}$ such that

$$
\frac{c\left(B_{w}\right)}{\operatorname{rk}(\mathcal{B})} \leq \widetilde{N}
$$

Since $\mathcal{H}^{0}\left(B_{w}\right)$ is a 0 -dimensional torsion sheaf, we have

$$
c\left(B_{w}\right)=-c(\mathcal{B})-\operatorname{ch}_{2}\left(\mathcal{H}^{0}\left(B_{w}\right)\right) \leq-c(\mathcal{B})=-\sum_{i=1}^{s} c\left(\mathcal{B}_{i}\right)
$$

where $\mathcal{B}_{1}, \ldots, \mathcal{B}_{s}$ are the usual $H N$-filtration quotients of $\mathcal{B}$ with respect to $\mu_{\omega}$ satisfying $\mu_{\omega}\left(\mathcal{B}_{1}\right)>\ldots>\mu_{\omega}\left(\mathcal{B}_{s}\right)$. To prove $(4.29)$, it suffices to show that each $c\left(\mathcal{B}_{i}\right) / \operatorname{rk}\left(\mathcal{B}_{i}\right)$ is bounded from below by a universal constant.

Finally, we analyze $\mathcal{B}_{i}$. Since $\mathcal{B}=\mathcal{H}^{-1}\left(B_{w}\right) \in \mathcal{F}_{(\omega, \beta \omega)}$, we see from the definition of $\mathcal{F}_{(\omega, \beta \omega)}$ that $\mathcal{B}_{i} \in \mathcal{F}_{(\omega, \beta \omega)}$ and $\mu_{\omega}\left(\mathcal{B}_{i}\right) \leq \beta \omega$. Let $\mathcal{E}=\mathcal{H}^{-1}(E)$. Let $\mathcal{F}$ (respectively, $\mathcal{G}$ ) be the image (respectively, cokernel) of the map $\mathcal{E} \rightarrow \mathcal{B}$ induced from (4.19). Combining the map $\mathcal{E} \rightarrow \mathcal{B}$ with the surjection $\mathcal{B} \rightarrow \mathcal{B}_{s}$, we obtain a map $f: \mathcal{E} \rightarrow \mathcal{B}_{s}$. Let $\widetilde{\mathcal{F}}$ be the image of $f$. If $\widetilde{\mathcal{F}}=0$, then we get an induced surjection $\mathcal{G} \cong \mathcal{B} / \mathcal{F} \rightarrow \mathcal{B}_{s}$. Since $\operatorname{Hom}\left(\mathcal{T}_{(\omega, \beta \omega)}, \mathcal{F}_{(\omega, \beta \omega)}\right)=0$, this is impossible by Lemma 2.2 (note that there exists an exact sequence of sheaves $0 \rightarrow \mathcal{G} \rightarrow \mathcal{H}^{0}\left(A_{w}\right) \rightarrow Q \rightarrow 0$ where $Q$ is a subsheaf of the 0 -dimensional torsion sheaf $\mathcal{H}^{0}(E)$ ). Thus, $\widetilde{\mathcal{F}} \neq 0$. Since $\mathcal{E}$ and $\mathcal{B}_{s}$ are $\mu_{\omega}$-semistable, $\mu_{\omega}(\mathcal{E}) \leq \mu_{\omega}(\widetilde{\mathcal{F}}) \leq \mu_{\omega}\left(\mathcal{B}_{s}\right)$. Therefore, we obtain $\mu_{\omega}(E)=\mu_{\omega}(\mathcal{E}) \leq$ $\mu_{\omega}\left(\mathcal{B}_{i}\right) \leq \beta \omega$ for every $i=1, \ldots, s$. By Lemma 3.7 (ii), each $c\left(\mathcal{B}_{i}\right) / \operatorname{rk}\left(\mathcal{B}_{i}\right)$ is bounded from below by a universal constant.

TheOREM 4.4. Let notations be from Subsect. 2.2, and let $\omega \in \operatorname{Num}(X)_{\mathbb{Q}}$. Fix a type $\mathfrak{t}=\left(r, c_{1}, c_{2}\right)$. Then there exists a positive number $M$, depending only on $\mathfrak{t}, \omega$ and $\beta$, such that $\overline{\mathfrak{M}}_{\mathbf{u}_{m}}(\mathfrak{t})=\overline{\mathfrak{M}}_{\Omega}(\mathfrak{t})$ for all $m \geq M$.

Proof. Follows immediately from Lemma 4.2 and Lemma 4.3.

TheOREM 4.5. Let $\beta, \omega \in \operatorname{Num}(X)_{\mathbb{Q}}$ with $\omega$ being ample, and let $\mathfrak{t}=\left(r, c_{1}, c_{2}\right)$.

(i) The set of mini-walls of type $(\mathfrak{t}, \beta, \omega)$ in $(0,+\infty)$ is locally finite. 
(ii) There exists a positive number $\widetilde{M}$, depending only on $\mathfrak{t}, \omega$ and $\beta$, such that there is no mini-wall of type $(\mathfrak{t}, \beta, \omega)$ in $[\widetilde{M},+\infty)$.

Proof. Part (i) is Proposition 3.10. To prove (ii), let $\mathbf{u}=e^{-(\beta+i \omega)}$ and $\widetilde{M}=1+M$ where $M$ is the positive number from Theorem 4.4. If $m_{0}$ is a mini-wall of type $(\mathfrak{t}, \beta, \omega)$ in $I=[\widetilde{M},+\infty)$, then by definition, $\phi\left(Z_{m_{0}}(A)\right)=\phi\left(Z_{m_{0}}(E)\right)$ where $E \in \overline{\mathfrak{M}}_{\mathbf{u}_{m_{1}}}(\mathfrak{t})$ for some $m_{1} \in I, E \notin \overline{\mathfrak{M}}_{\mathbf{u}_{m_{2}}}(\mathfrak{t})$ for some $m_{2} \in I$, and $A$ is the leading HN-filtration component of $E$ with respect to $\left(Z_{m_{2}}, \mathcal{P}_{m_{2}}\right)$. In particular, $\overline{\mathfrak{M}}_{\mathbf{u}_{m_{1}}}(\mathfrak{t}) \neq \overline{\mathfrak{M}}_{\mathbf{u}_{m_{2}}}(\mathfrak{t})$. This contradicts Theorem 4.4 since $m_{1}, m_{2} \geq \widetilde{M}>M$. $\square$

Corollary 4.6. Let $\beta, \omega \in \operatorname{Num}(X)_{\mathbb{Q}}$ with $\omega$ being ample. Fix a numerical type $\mathfrak{t}=\left(r, c_{1}, c_{2}\right)$ and an interval $I=[a,+\infty)$ with $a>0$. Then there exists a finite subset $\left\{m_{0}^{(1)}, \ldots, m_{0}^{(n)}\right\} \subset I$, possibly empty, such that $\overline{\mathfrak{M}}_{\mathbf{u}_{m_{1}}}(\mathfrak{t})=\overline{\mathfrak{M}}_{\mathbf{u}_{m_{2}}}(\mathfrak{t})$ whenever $m_{1}$ and $m_{2}$ are contained in the same connected component of $I-\left\{m_{0}^{(1)}, \ldots, m_{0}^{(n)}\right\}$.

Proof. Let $\widetilde{M}$ be from Theorem 4.5 (ii). If $\widetilde{M} \leq a$, then the result is true by Theorem 4.5 (ii) and Lemma 3.4. If $\widetilde{M}>a$, then let $\left\{m_{0}^{(1)}, \ldots, m_{0}^{(n-1)}\right\}$ be the finite set of mini-walls of type $(\mathfrak{t}, \beta, \omega)$ in $[a, \widetilde{M}]$. Letting $m_{0}^{(n)}=\widetilde{M}$, we are done.

5. Identify $\overline{\mathfrak{M}}_{\Omega}(\mathfrak{t})$ with Gieseker and Uhlenbeck moduli spaces. Fix a numerical type $\mathfrak{t}=\left(r, c_{1}, c_{2}\right)$. In this section, we will compare the spaces $\overline{\mathfrak{M}}_{\Omega}(\mathfrak{t})$ with the Gieseker/Simpson and Uhlenbeck spaces where $\Omega$ comes from Subsect. 2.2. The results here are similar to those in Sect. 4 of [LQ].

In view of Lemma 2.10 and Lemma 2.11, we will assume that $r \neq 0$. We will further assume that $\omega \in \operatorname{Num}(X)_{\mathbb{Z}}$ and $\operatorname{gcd}\left(r, c_{1} \omega\right)=1$. These conditions ensure that $\omega$ does not lie on any wall of type $\mathfrak{t}$ and that every $\mu_{\omega}$-semistable sheaf is automatically $\mu_{\omega}$-stable.

Lemma 5.1. Let $\Omega=(\omega, \rho, p, U)$ be from Subsect. 2.2 with $\omega \in \operatorname{Num}(X)_{\mathbb{Z}}$. Fix a numerical type $\mathfrak{t}=\left(r, c_{1}, c_{2}\right)$ with $r>0, c_{1} \omega / r>\beta \omega$ and $\operatorname{gcd}\left(r, c_{1} \omega\right)=1$. Then, every object in $\overline{\mathfrak{M}}_{\Omega}(\mathfrak{t})$ is $\left(Z_{\Omega}, \mathcal{P}_{\Omega}\right)$-stable, and $\overline{\mathfrak{M}}_{\Omega}(\mathfrak{t})=\overline{\mathfrak{M}}_{\omega}(\mathfrak{t})$.

Proof. Let $E \in \overline{\mathfrak{M}}_{\Omega}(\mathfrak{t})$. By Lemma 2.4, $E$ is a $\mu_{\omega}$-semistable torsion free sheaf. Since $\operatorname{gcd}\left(r, c_{1} \omega\right)=1, E$ must be $\mu_{\omega}$-stable. In particular, $E \in \overline{\mathfrak{M}}_{\omega}(\mathfrak{t})$.

Conversely, let $E \in \overline{\mathfrak{M}}_{\omega}(\mathfrak{t})$. Then $E$ is $\mu_{\omega}$-stable since $\operatorname{gcd}\left(r, c_{1} \omega\right)=1$. Since $\mu_{\omega}(E)=c_{1} \omega / r>\beta \omega, E \in \mathcal{P}_{\Omega}((0,1])=\mathcal{A}_{(\omega, \beta \omega)}^{\sharp}$. Let $A$ be any proper sub-object of $E$ in $\mathcal{P}_{\Omega}((0,1])$, and let $B=E / A$. Then we have the exact sequence $0 \rightarrow A \rightarrow E \rightarrow$ $B \rightarrow 0$ in $\mathcal{P}_{\Omega}((0,1])$. So $A$ is a sheaf in $\mathcal{T}_{(\omega, \beta \omega)}$ and sits in

$$
0 \rightarrow \mathcal{H}^{-1}(B) \rightarrow A \rightarrow E \rightarrow \mathcal{H}^{0}(B) \rightarrow 0 .
$$

It follows that $A$ is torsion free with $\mu_{\omega}(A)>\beta \omega$. If $\mathcal{H}^{-1}(B) \neq 0$, then $\mathcal{H}^{-1}(B) \in$ $\mathcal{F}_{(\omega, \beta \omega)}$. So $\mu_{\omega}\left(\mathcal{H}^{-1}(B)\right) \leq \beta \omega<\mu_{\omega}(A)$. Thus the image $\mathcal{G}$ of $A \rightarrow E$ is not zero. Since $E$ is $\mu_{\omega}$-stable, we conclude that $\mu_{\omega}(A)<\mu_{\omega}(\mathcal{G}) \leq \mu_{\omega}(E)$. By $(2.7)$, $\phi\left(Z_{\Omega}(E)(m)\right)>\phi\left(Z_{\Omega}(A)(m)\right)$ for all $m \gg 0$. If $\mathcal{H}^{-1}(B)=0$, then we have an exact sequence $0 \rightarrow A \rightarrow E \rightarrow B \rightarrow 0$ of sheaves. Since $A$ is a proper subsheaf of $E$, $\mu_{\omega}(A)<\mu_{\omega}(E)$. So again $\phi\left(Z_{\Omega}(E)(m)\right)>\phi\left(Z_{\Omega}(A)(m)\right)$ for all $m \gg 0$. Therefore, $E$ is $\left(Z_{\Omega}, \mathcal{P}_{\Omega}\right)$-stable. In particular, $E \in \overline{\mathfrak{M}}_{\Omega}(\mathfrak{t})$.

Lemma 5.2. Let $\Omega=(\omega, \rho, p, U)$ be from Subsect. 2.2 with $\omega \in \operatorname{Num}(X)_{\mathbb{Z}}$. Fix a numerical type $\mathfrak{t}=\left(r, c_{1}, c_{2}\right)$ with $r<0, c_{1} \omega / r<\beta \omega$ and $\operatorname{gcd}\left(r, c_{1} \omega\right)=1$. Let 
$\tilde{\mathfrak{t}}=\left(-r, c_{1}, c_{1}^{2}-c_{2}\right)$. Then, every object in $\overline{\mathfrak{M}}_{\Omega}(\mathfrak{t})$ is $\left(Z_{\Omega}, \mathcal{P}_{\Omega}\right)$-stable, and $E \in \overline{\mathfrak{M}}_{\Omega}(\mathfrak{t})$ if and only if $E=(\widetilde{E})^{\vee}[1]$ for some $\widetilde{E} \in \overline{\mathfrak{M}}_{\omega}(\tilde{\mathfrak{t}})$.

Proof. Let $E \in \overline{\mathfrak{M}}_{\Omega}(\mathfrak{t})$. Then, $\left(c_{1}(E) \cdot \omega-\operatorname{rk}(E) \beta \omega\right)=c_{1} \omega-r \beta \omega>0$. By (2.5), $\phi\left(Z_{\Omega}(E)(m)\right)<1$ for all $m>0$. So $E$ does not have any sub-objects in $\mathcal{P}_{\Omega}((0,1])$ which are 0 -dimensional torsion sheaves. By Lemma $2.4, \mathcal{H}^{-1}(E)$ is a torsion free $\mu_{\omega}$-stable sheaf and $\mathcal{H}^{0}(E)$ is a 0 -dimensional torsion sheaf. Note that $\mathcal{H}^{-1}(E)$ must be locally free (otherwise, the 0-dimensional torsion sheaf $\left(\mathcal{H}^{-1}(E)\right)^{* *} / \mathcal{H}^{-1}(E)$ would be a sub-object of $E$ in $\left.\mathcal{P}_{\Omega}((0,1])\right)$. By the Lemma 3.4 in [ABL], $E=(\widetilde{E})^{\vee}[1]$ for some torsion free sheaf $\widetilde{E}$. A direct computation shows that $\mathfrak{t}(\widetilde{E})=\tilde{\mathfrak{t}}$. Since $(\widetilde{E})^{*}=\mathcal{H}^{-1}(E)$ is $\mu_{\omega}$-stable, so is $\widetilde{E}$. In particular, $\widetilde{E} \in \overline{\mathfrak{M}}_{\omega}(\tilde{\mathfrak{t}})$.

Conversely, let $E=(\widetilde{E})^{\vee}[1]$ for some $\widetilde{E} \in \overline{\mathfrak{M}}_{\omega}(\tilde{\mathfrak{t}})$. Then $\mathcal{H}^{0}(E)=\mathcal{E} x t^{1}\left(\widetilde{E}, \mathcal{O}_{X}\right)$ is a 0 -dimensional torsion sheaf, and $\mathcal{H}^{-1}(E)=(\widetilde{E})^{*}$ is locally free and $\mu_{\omega}$-stable with $\mu_{\omega}\left((\widetilde{E})^{*}\right)=\left(-c_{1}\right) \omega /(-r)<\beta \omega$. So $E \in \mathcal{P}_{\Omega}((0,1])$. Let $A$ be any proper sub-object of $E$ in $\mathcal{P}_{\Omega}((0,1])$, and let $B=E / A$. Then we have the exact sequence $0 \rightarrow A \rightarrow E \rightarrow B \rightarrow 0$ in $\mathcal{P}_{\Omega}((0,1])$ and an exact sequence of sheaves

$$
0 \rightarrow \mathcal{H}^{-1}(A) \rightarrow(\widetilde{E})^{*} \rightarrow \mathcal{H}^{-1}(B) \rightarrow \mathcal{H}^{0}(A) \rightarrow \mathcal{H}^{0}(E) \rightarrow \mathcal{H}^{0}(B) \rightarrow 0 .
$$

Let $\mathcal{F}$ and $\mathcal{G}$ be the image and cokernel of $(\widetilde{E})^{*} \rightarrow \mathcal{H}^{-1}(B)$ respectively.

We claim that $A$ does not have any sub-object $Q$ in $\mathcal{P}_{\Omega}((0,1])$ which is a 0 dimensional torsion sheaf. Indeed, if such a $Q$ exists, then $Q$ is a sub-object of $E=(\widetilde{E})^{\vee}[1]$ in $\mathcal{P}_{\Omega}((0,1])$. In particular, there exists a point $x \in X$ such that $\mathcal{O}_{x}$ is a sub-object of $E=(\widetilde{E})^{\vee}[1]$ in $\mathcal{P}_{\Omega}((0,1])$. This leads to a contradiction:

$$
\begin{aligned}
0 & \neq \operatorname{Hom}_{\mathcal{P}_{\Omega}((0,1])}\left(\mathcal{O}_{x},(\widetilde{E})^{\vee}[1]\right) \cong \operatorname{Hom}_{\mathcal{D}^{b}(X)}\left(\widetilde{E}[-1], \mathcal{O}_{x}^{\vee}\right) \\
& =\operatorname{Hom}_{\mathcal{D}^{b}(X)}\left(\widetilde{E}[-1], \mathcal{O}_{x}[-2]\right) \cong \operatorname{Ext}_{\operatorname{Coh}(X)}^{-1}\left(\widetilde{E}, \mathcal{O}_{x}\right)=0
\end{aligned}
$$

where we have used the fact that $\mathcal{O}_{x}^{\vee}$, the derived dual of $\mathcal{O}_{x}$, is equal to $\mathcal{O}_{x}[-2]$.

If $\mathcal{H}^{-1}(A)=0$, then $A$ is a sheaf in $\mathcal{T}_{(\omega, \beta \omega)}$. If $A$ is a 1 -dimensional torsion sheaf, then we see from $(2.7)$ that $\phi\left(Z_{\Omega}(E)(m)\right)>\phi\left(Z_{\Omega}(A)(m)\right)$ for all $m \gg 0$. Assume that $A$ is not a 1-dimensional torsion sheaf. By the preceding paragraph, $A$ can not be a 0 -dimensional torsion sheaf. So $\operatorname{rk}(A)>0$ and $\mu_{\omega}(A)>\beta \omega>\mu_{\omega}\left((\widetilde{E})^{*}\right)=\mu_{\omega}(E)$. By $(2.7), \phi\left(Z_{\Omega}(E)(m)\right)>\phi\left(Z_{\Omega}(A)(m)\right)$ for all $m \gg 0$.

If $\mathcal{B}:=\mathcal{H}^{-1}(B)=0$, then $B$ is a 0 -dimensional torsion sheaf and $\phi\left(Z_{\Omega}(B)(m)\right)=$ 1 for all $m>0$. By $(2.5), \phi\left(Z_{\Omega}(E)(m)\right)<\phi\left(Z_{\Omega}(B)(m)\right)$ for all $m>0$.

In the following, assume that $\mathcal{H}^{-1}(A) \neq 0$ and $\mathcal{B} \neq 0$. Then $\mathcal{B} \in \mathcal{F}_{(\omega, \beta \omega)}$ is torsion free with $\mu_{\omega}(\mathcal{B}) \leq \beta \omega$. If $\mathcal{F}=0$, then $\beta \omega \geq \mu_{\omega}(\mathcal{B})=\mu_{\omega}\left(\mathcal{H}^{0}(A)\right)$ since $\mathcal{H}^{0}(A) / \mathcal{B}$ is a subsheaf of the 0 -dimensional torsion sheaf $\mathcal{H}^{0}(E)$. This contradicts $\mathcal{H}^{0}(A) \in \mathcal{T}_{(\omega, \beta \omega)}$. Assume that $\mathcal{F} \neq 0$. Then $\mathcal{F}$ is a proper quotient of $(\widetilde{E})^{*}$. Since $(\widetilde{E})^{*}$ is $\mu_{\omega^{-}}$stable, $\mu_{\omega}\left((\widetilde{E})^{*}\right)<\mu_{\omega}(\mathcal{F})$. If $\operatorname{rk}(\mathcal{G})=0$, then we see from the exact sequence $0 \rightarrow \mathcal{F} \rightarrow \mathcal{B} \rightarrow \mathcal{G} \rightarrow 0$ that $\mu_{\omega}(\mathcal{B}) \geq \mu_{\omega}(\mathcal{F})>\mu_{\omega}\left((\widetilde{E})^{*}\right)$; if $\operatorname{rk}(\mathcal{G})>0$, then $\mu_{\omega}(\mathcal{G})=\mu_{\omega}\left(\mathcal{H}^{0}(A)\right)>\beta \omega$ since $\mathcal{H}^{0}(A) / \mathcal{G}$ is a subsheaf of $\mathcal{H}^{0}(E)$. Since $\mu_{\omega}(\mathcal{B}) \leq \beta \omega$, we have $\mu_{\omega}(\mathcal{G})>\mu_{\omega}(\mathcal{B})>\mu_{\omega}(\mathcal{F})>\mu_{\omega}\left((\widetilde{E})^{*}\right)$. In either case, $\mu_{\omega}(\mathcal{B})>\mu_{\omega}\left((\widetilde{E})^{*}\right)$. Hence $\mu_{\omega}(B)>\mu_{\omega}(E)$. By $(2.7), \phi\left(Z_{\Omega}(E)(m)\right)<\phi\left(Z_{\Omega}(B)(m)\right)$ for all $m \gg 0$. This proves that $E$ is $\left(Z_{\Omega}, \mathcal{P}_{\Omega}\right)$-stable.

Lemma 5.3. Let $\Omega=(\omega, \rho, p, U)$ be from Subsect. 2.2 with $\omega \in \operatorname{Num}(X)_{\mathbb{Z}}$. Fix a numerical type $\mathfrak{t}=\left(r, c_{1}, c_{2}\right)$ with $r<0, c_{1} \omega / r=\beta \omega$ and $\operatorname{gcd}\left(r, c_{1} \omega\right)=1$. 
(i) If $\widetilde{E} \in \mathfrak{M}_{\omega}\left(-r, c_{1}, c_{1}^{2}-\left(c_{2}+i\right)\right)$ for some $i \geq 0$ and $Q$ is a length-i 0 dimensional torsion sheaf, then $(\widetilde{E})^{*}[1] \oplus Q \in \overline{\mathfrak{M}}_{\Omega}(\mathfrak{t})$.

(ii) If $E \in \overline{\mathfrak{M}}_{\Omega}(\mathfrak{t})$, then $E$ is $S$-equivalent to $(\widetilde{E})^{*}[1] \oplus Q$ where $Q$ is a length-i 0 -dimensional torsion sheaf and $\widetilde{E} \in \mathfrak{M}_{\omega}\left(-r, c_{1}, c_{1}^{2}-\left(c_{2}+i\right)\right)$.

Proof. (i) Recall from Definition 2.9 (iv) that $\widetilde{E}$ is locally free. Note that the numerical type of $(\widetilde{E})^{*}[1] \oplus Q$ is $\mathfrak{t}$, and $(\widetilde{E})^{*}[1], Q \in \mathcal{P}_{\Omega}((0,1])=\mathcal{A}_{(\omega, \beta \omega)}^{\sharp}$ with

$$
\phi\left(Z_{\Omega}\left((\widetilde{E})^{*}[1]\right)(m)\right)=\phi\left(Z_{\Omega}(Q)(m)\right)=1
$$

for all $m>0$. Also, $Q$ is $\left(Z_{\Omega}, \mathcal{P}_{\Omega}\right)$-semistable. A slight modification of the proof of Lemma 5.2 shows that $(\widetilde{E})^{*}[1]$ is $\left(Z_{\Omega}, \mathcal{P}_{\Omega}\right)$-stable as well. It follows that $(\widetilde{E})^{*}[1] \oplus Q$ is $\left(Z_{\Omega}, \mathcal{P}_{\Omega}\right)$-semistable. Therefore, we have $(\widetilde{E})^{*}[1] \oplus Q \in \overline{\mathfrak{M}}_{\Omega}(\mathfrak{t})$.

(ii) Let $E \in \overline{\mathfrak{M}}_{\Omega}(\mathfrak{t})$. Since $c_{1} \omega / r=\beta \omega, \phi\left(Z_{\Omega}(E)(m)\right)=1$ for all $m>0$. By Lemma 2.4, $\mathcal{H}^{0}(E)$ is a 0 -dimensional torsion sheaf, and $\mathcal{H}^{-1}(E)$ is a torsion free $\mu_{\omega}$-stable sheaf. From the exact sequence $0 \rightarrow \mathcal{H}^{-1}(E)[1] \rightarrow E \rightarrow \mathcal{H}^{0}(E) \rightarrow 0$ in $\mathcal{P}_{\Omega}((0,1])$, we see that $E$ is $S$-equivalent to $\mathcal{H}^{-1}(E)[1] \oplus \mathcal{H}^{0}(E)$. Thus to prove our result, we may assume that $E=A[1]$ for some torsion free $\mu_{\omega}$-stable sheaf $A$ with $\mu_{\omega}(A)=\beta \omega$. We have the canonical exact sequence $0 \rightarrow A \rightarrow A^{* *} \rightarrow Q \rightarrow 0$ where $Q$ is a 0 -dimensional torsion sheaf. It gives rise to an exact sequence

$$
0 \rightarrow Q \rightarrow A[1] \rightarrow A^{* *}[1] \rightarrow 0
$$

in $\mathcal{P}_{\Omega}((0,1])$. Hence $E=A[1]$ is $S$-equivalent to $A^{* *}[1] \oplus Q$.

Theorem 5.4. Let $\Omega=(\omega, \rho, p, U)$ be from Subsect. 2.2 with $\omega \in \operatorname{Num}(X)_{\mathbb{Z}}$. Fix a numerical type $\mathfrak{t}=\left(r, c_{1}, c_{2}\right)$ with $\operatorname{gcd}\left(r, c_{1} \omega\right)=1$. Let $\tilde{\mathfrak{t}}=\left(-r, c_{1}, c_{1}^{2}-c_{2}\right)$.

(i) If $r>0$, then $\overline{\mathfrak{M}}_{\Omega}(\mathfrak{t}) \cong \overline{\mathfrak{M}}_{\omega}(\mathfrak{t})$.

(ii) If $r<0$ and $c_{1} \omega / r<\beta \omega$, then $\overline{\mathfrak{M}}_{\Omega}(\mathfrak{t}) \cong \overline{\mathfrak{M}}_{\omega}(\tilde{\mathfrak{t}})$.

(iii) If $r<0$ and $c_{1} \omega / r=\beta \omega$, then $\overline{\mathfrak{M}}_{\Omega}(\mathfrak{t}) \cong \overline{\mathfrak{U}}_{\omega}(\tilde{\mathfrak{t}})$.

Proof. Follows from Lemma 5.1, Lemma 5.2 and Lemma 5.3. Note from Lemma 2.4 that in (i), we automatically have $c_{1} \omega / r>\beta \omega$ if $\overline{\mathfrak{M}}_{\Omega}(\mathfrak{t}) \neq \emptyset$. Note also that in (iii), if $E \in \overline{\mathfrak{M}}_{\Omega}(\mathfrak{t})$ is $S$-equivalent to $(\widetilde{E})^{*}[1] \oplus Q$ where $\widetilde{E} \in \mathfrak{M}_{\omega}\left(-r, c_{1}, c_{1}^{2}-\left(c_{2}+i\right)\right)$ for some $i \geq 0$ and $Q$ is a length- $i$-dimensional torsion sheaf, then we map $E$ to

$$
\left(\widetilde{E}, \sum_{x \in X} h^{0}\left(X, Q_{x}\right) \cdot x\right)
$$

in $\mathfrak{M}_{\omega}\left(-r, c_{1}, c_{1}^{2}-\left(c_{2}+i\right)\right) \times \operatorname{Sym}^{i}(X) \subset \overline{\mathfrak{U}}_{\omega}(\tilde{\mathfrak{t}})($ see $(2.14))$.

\section{REFERENCES}

[ABL] D. Arcara, A. Bertram, And M. Lieblich, Bridgeland-stable moduli spaces for K-trivial surfaces, J. Euro. Math. Soc., 15 (2013), pp. 1-38.

[Bay] A. BAYER, Polynomial Bridgeland stability conditions and the large volume limit, Geom. Topol., 13 (2009), pp. 2389-2425.

[Bri1] T. Bridgeland, Stability conditions on triangulated categories, Ann. Math., 100 (2007), pp. $317-346$

[Bri2] T. BRIDGeland, Stability conditions on K3 surfaces, Duke Math. J., 141 (2008), pp. 241291.

[CP] J. Collins And A. Polishchuk, Gluing stability conditions, Adv. Theor. Math. Phys., 14 (2010), pp. 563-607. 
[Dou] M. R. Douglas, D-branes, categories and $N=1$ supersymmetry. Strings, branes, and M-theory, J. Math. Phys., 42 (2001), pp. 2818-2843.

[HMS] D. Huybrechts, E. Macri, AND P. Stellari, Stability conditions for generic K3 categories, Compos. Math., 144 (2008), pp. 134-162.

[Ina] M. InABA, Moduli of stable objects in a triangulated category, J. Math. Soc. Japan, 62 (2010), pp. 395-429.

[Kaw] K. KaWATANI, Stability conditions and $\mu$-stable sheaves on K3 surfaces with Picard number one, preprint. arXiv: 1005.3877.

[KS] M. Kontsevich And Y. Soibelman, Stability structures, motivic Donaldson-Thomas invariants and cluster transformations, preprint.

[Li1] J. LI, Algebraic geometric interpretation of Donaldson's polynomial invariants, J. Differ. Geom., 37 (1993), pp. 417-466.

[Li2] J. LI, Hermitian-Yang-Mills connections on Kähler manifolds, in Geometry and Analysis (Vol II), ALM, 18 (2010), pp. 81-102.

[LQ] W.-P. Li AND Z. QIN, Polynomial Bridgeland stability for the derived category of sheaves on surfaces, Communications in Analysis and Geometry, 19 (2011), pp. 31-52.

[Lie] M. Lieblich, Moduli of complexes on a proper morphism, J. Algebraic Geom., 15 (2006), pp. 175-206.

[Lo1] J. LO, Moduli spaces of PT-stable objects, Ph.D. Thesis, Stanford University, 2010.

[Lo2] J. Lo, Moduli of PT-semistable objects I, J. Algebra, 339 (2011), pp. 203-222.

[Lo3] J. Lo, Moduli of PT-semistable objects II, Trans. Amer. Math. Soc. (to appear).

[Mac] E. MACRI, Stability conditions on curves, Math. Res. Lett., 14 (2007), pp. 657-672.

[Mor] J. W. Morgan, Comparison of the Donaldson polynomial invariants with their algebrogeometric analogues, Topology, 32 (1993), pp. 449-488.

[Ohk] R. Ohkawa, Moduli of Bridgeland semistable objects on $\mathbb{P}^{2}$, Kodai Math. J., 33 (2010), pp. 329-366.

[Oka] S. OkADA, Stability manifold of $\mathbb{P}^{1}$, J. Algebraic Geom., 15 (2006), pp. 487-505.

[Qin] Z. QIN, Equivalence classes of polarizations and moduli spaces of sheaves, J. Differ. Geom., 37 (1993), pp. 397-415.

[Tod1] Y. ToDA, Moduli stacks and invariants of semistable objects on K3 surfaces, Adv. Math., 217 (2008), pp. 2736-2781.

[Tod2] Y. TodA, Limit stable objects on Calabi-Yau 3-folds, Duke Math. J., 149 (2009), pp. 157208.

[Tod3] Y. TodA, Stability conditions and Calabi-Yau fibrations, J. Algebraic Geom., 18 (2009), pp. 101-133.

[Yos1] K. YoshiokA, Stability and twisted Fourier-Mukai transform, II, Compositio Math., 145 (2009), pp. 112-142.

[Yos2] K. YoshiokA, Perverse coherent sheaves and Fourier-Mukai transforms on surfaces, preprint. arXiv:1003.2522.

[Yos3] K. YoshiokA, A few remarks on the paper "Perverse coherent sheaves and Fourier-Mukai transforms on surfaces", private communications, 2011. 